\title{
Mechanosensation by endothelial PIEZO1 is required for leukocyte diapedesis
}

\section{Stefan Offermanns ( $\nabla$ stefan.offermanns@mpi-bn.mpg.de )}

Max Planck Institute for Heart and Lung Research https://orcid.org/0000-0001-8676-6805

\section{ShengPeng Wang}

Cardiovascular Research Center, School of Basic Medical Sciences, Xi'an Jiaotong University Health

Science Center, Yanta District, Xi'an, China https://orcid.org/0000-0002-9030-1821

\section{Yue Shi}

Cardiovascular Research Center, School of Basic Medical Sciences, Xi'an Jiaotong University Health

Science Center

\section{Tanja Moeller}

Max Planck Institute of Molecular Biomedicine, Muenster, Germany

\section{Rebekka Stegmeyer}

Max Planck Institute of Molecular Biomedicine, Muenster, Germany

\section{Boris Strilic}

Max Planck Institute for Heart and Lung Research

\section{Liran Xu}

Cardiovascular Research Center, School of Basic Medical Sciences, Xi'an Jiaotong University

\section{Zuyi Yuan}

Xian Jiaotong University

\section{Nina Wettschureck}

Department of Pharmacology, Max Planck Institute for Heart and Lung Research, Bad Nauheim, 61231, Germany

\section{Dietmar Vestweber}

Max Planck Institute for Molecular Biomedicine https://orcid.org/0000-0002-3517-732X

\section{Biological Sciences - Article}

Keywords: leukocyte diapedesis, inflammation

Posted Date: February 12th, 2021

DOl: https://doi.org/10.21203/rs.3.rs-208666/v1

License: (c) (i) This work is licensed under a Creative Commons Attribution 4.0 International License. 



\title{
Mechanosensation by endothelial PIEZO1 is required for leukocyte diapedesis
}

\author{
ShengPeng Wang ${ }^{1,2,{ }^{*}}$, Yue Shi ${ }^{1,2}$, Tanja Möller $^{3}$, Rebekka I. Stegmeyer $^{3}$,
} Boris Strilic ${ }^{2}$, Liran Xu ${ }^{1}$, Zuyi Yuan ${ }^{1}$, Nina Wettschureck ${ }^{2,4,5}$, Dietmar Vestweber ${ }^{3}$, Stefan Offermanns ${ }^{2,4,5,{ }^{*}}$

${ }^{1}$ Department of Cardiology, First Affiliated Hospital, Cardiovascular Research Center, School of Basic Medical Sciences, Xi'an Jiaotong University, No.277 West Yanta Road, Yanta District, Xi'an, China

${ }^{2}$ Max Planck Institute for Heart and Lung Research, Department of Pharmacology, Ludwigstr. 43, 61231 Bad Nauheim, Germany

${ }^{3}$ Department of Vascular Cell Biology, Max Planck Institute of Molecular Biomedicine, Muenster, Germany

${ }^{4}$ Center for Molecular Medicine, Goethe University Frankfurt, Theodor-Stern-Kai 7, 60590 Frankfurt, Germany

${ }^{5}$ Cardiopulmonary Institute, 61231 Bad Nauheim, Germany 9

"Correspondence: S.P.W. (shengpeng.wang@xitu.edu.cn) and

S.O. (stefan.offermanns@mpi-bn.mpg.de) 


\section{Summary}

2 The extravasation of leukocytes is a critical step during inflammation which requires

3 the localized opening of the endothelial barrier ${ }^{1-3}$. This process is initiated by the

4 close interaction of leukocytes with various adhesion molecules such as intercellular

5 adhesion molecule-1 (ICAM-1) on the surface of endothelial cells ${ }^{4-6}$. It is still unclear

6 how these initial processes induce downstream signaling events resulting in the

7 opening of inter-endothelial junctions to allow leukocyte diapedesis. Here we show

8 that mechanical forces induced by leukocyte-induced clustering of ICAM-1 and fluid

9 shear stress exerted by the flowing blood synergistically activate the

10 mechanosensitive cation channel PIEZO1 in endothelial cells. In human and mouse

11 endothelial cells exposed to low flow, PIEZO1 mediates leukocyte-induced increases

12 in $\left[\mathrm{Ca}^{2+}\right]_{i}$ and activation of downstream signaling events including phosphorylation of

13 SRC, PYK2 and myosin light chain (MLC) leading to endothelial barrier opening.

14 Mice with endothelium-specific loss of Piezo1 show decreased leukocyte 15 extravasation in different inflammation models. We found that actin polymerization 16 and actomyosin contraction induced by ICAM-1 clustering synergistically with fluid 17 shear stress increase endothelial plasma membrane tension to activate PIEZO1. Our 18 data reveal a mechanism by which leukocytes and the hemodynamic 19 microenvironment synergize to mechanically activate endothelial PIEZO1 and 20 subsequent downstream signaling to initiate leukocyte diapedesis. 


\section{$1 \quad$ Main Text}

2 The endothelial cell layer is a tight barrier for cells in the circulation. However, during

3 inflammation leukocytes are able to transmigrate the endothelium and to extravasate

4 in the perivascular space, a process which involves a well-coordinated cascade of 5 events. This includes initial leukocyte capture and rolling, firm adhesion, crawling, 6 which are then followed by breaching of the endothelial barrier and the 7 extravasation ${ }^{7,8}$. The molecular mechanisms that control and mediate the initial 8 interactions between leukocytes and endothelial cells are well-characterized and 9 involve interactions between endothelial selectins and glycoproteins of leukocytes during capture and rolling steps, whereas arrest, firm adhesion and crawling are

11 mediated mainly by integrins on leukocytes which bind to endothelial intercellular and 12 vascular cell adhesion molecules (ICAM-1 and VCAM-1) and induce their 13 clustering ${ }^{5,8,9}$. How these initial processes are linked to the opening of the endothelial barrier, which requires the remodeling of endothelial adherens junction as well as endothelial cell contraction ${ }^{2,3,6,10,11}$ is, however, poorly understood.

Opening of endothelial junctions and endothelial cell contraction during leukocyte transmigration requires activation of endothelial signaling pathways, and several studies have shown that leukocytes induce an increase in the cytosolic $\mathrm{Ca}^{2+}$ concentration in endothelial cells ${ }^{12-16}$. This calcium signal is not necessary for leukocyte adhesion but is required to induce transendothelial migration ${ }^{12-14}$. ICAM-1

21 has been shown to be involved in lymphocyte-induced $\mathrm{Ca}^{2+}$ transients in endothelial 22 cells $^{14}$, and more recently, the transient receptor potential (TRP) channel C6 (TRPC6) 23 was shown to be involved in endothelial calcium transients induced by neutrophils 24 and for their transendothelial migration ${ }^{17}$, but how leukocytes induce endothelial $\mathrm{Ca}^{2+}$ 25 transients is still unclear. 
The Piezo proteins PIEZO1 and PIEZO2 are mechanically activated cation channels that form homotrimeric complexes ${ }^{18-20}$, which are sufficient to mediate mechanically induced currents ${ }^{18}$. PIEZO1 has been shown to be gated directly by changes in membrane tension ${ }^{21,22}$ and to mediate multiple cellular functions including endothelial flow sensing ${ }^{23,24}$.

\section{PIEZO1 is required for leukocyte transendothelial migration in vitro}

In a screen to identify endothelial transmembrane proteins involved in the transendothelial migration of leukocytes, we identified the mechanosensitive cation channel PIEZO1 (Fig. 1a). The siRNA-mediated knock-down of PIEZO1 in human umbilical venous endothelial cells (HUVECs) or in the mouse brain endothelial cell line bEnd.3 strongly reduced endothelial transmigration of polymorphonuclear leukocytes (PMNs) and peripheral blood mononuclear cells (PBMCs) (Fig. 1b, Extended Data Fig. 1a,b). Similarly, PMN transmigration through mouse lung endothelial cells (MLECs) from mice with endothelium-specific loss of Piezo1 (ECPiezo1-KO) was strongly reduced compared to wild-type MLECs (Fig. 1c). Basal expression of VE-cadherin and PECAM-1 as well as TNFa-induced expression of ICAM-1 was not affected by loss of PIEZO1 (Extended Data Fig. 1c). Both, PMN transmigration of human and murine endothelial cells could be stimulated by Yoda1, an activator of PIEZO1, and this effect was not seen after knock-down of PIEZO1 in endothelial cells (Fig. 1c, Extended Data Fig. 1b,d,e). PMN rolling on and adhesion to endothelial cells was not affected by loss of endothelial Piezo1 expression (Fig. 1b, Extended Data Fig. 1a), and endothelial barrier function analyzed by measuring the electrical impedance of the endothelial cell layer in vitro or by determining the permeability of the endothelial layer for FITC-labelled dextran in vivo was not affected by loss of PIEZO1 (Extended Data Fig. 1f,g). 


\section{Endothelial PIEZO1 is critically involved in leukocyte extravasation in vivo}

2 To study leukocyte extravasation in vivo, we injected TNFa into the peritoneal cavity

3 and determined the number of $\mathrm{CD} 11 \mathrm{~b}^{+} / \mathrm{Ly} 6 \mathrm{G}^{+}$myeloid cells in the peritoneal cavity 6

4 hours later. While TNFa induced a significant influx of cells into the peritoneal cavity

5 of wild-type mice compared to untreated controls, the effect of TNFa was strongly

6 reduced in EC-Piezo1-KO mice (Fig. 1d). We then studied the role of endothelial

7 PIEZO1 in a model of acute dermatitis of the ear by applying croton oil to the ear

8 surface. Six hours later, when analyzing postcapillary venules characterized by a

9 diameter of $20-30 \mu \mathrm{m}$, the primary site of leukocyte extravasation, we found that the majority of neutrophils had completed extravasation and were found in the perivascular space in wild-type mice, whereas about $25-30 \%$ of the leukocytes were found in the lumen of vessels (Fig. 1e-g). However, in EC-Piezo1-KO mice, a significantly reduced portion of leukocytes had completed extravasation, and the majority, about $70 \%$ of cells, showed arrest at the luminal surface of the endothelium (Fig. 1e,g), suggesting that they adhered to the endothelium but were not able to initiate the process of endothelial transmigration. Also intravital microscopy of the cremaster of EC-Piezo1-KO mice revealed a reduced extravasation of neutrophils compared to wild-type animals after intrascrotal injection of IL-1 $\beta$ (Fig. $1 \mathrm{~h}$ ). Hemodynamic parameters were similar in both mouse types, and there was no significant different in leukocyte rolling and adhesion within venules (Extended Data Fig. 2a-e). Also basal extravasation of Evans blue and extravasation after subcutaneous injection of histamine or VEGF were indistinguishable between wildtype and EC-Piezo1-KO mice (Fig. 1i), indicating that vascular permeability was unchanged. Expression of genes encoding proteins involved in endothelial functions was not changed in endothelial cells from EC-Piezo1-KO mice (Extended Data Fig. $2 f, g)$. 
1 Leukocytes and low flow synergistically induce downstream signaling via

2 PIEZO1

3 Since increases in $\left[\mathrm{Ca}^{2+}\right]_{\mathrm{i}}$ are involved in the initiation of leukocyte transendothelial

4 migration and since leukocyte diapedesis occurs in the presence of low flow in vivo,

5 we studied leukocyte-induced increases in endothelial cytosolic $\mathrm{Ca}^{2+}$ in the absence

6 and presence of flow at a low shear rate $\left(1.2\right.$ dynes $\left./ \mathrm{cm}^{2}\right)$. In control HUVECs loaded

7 with Fluo4, low flow or addition of human neutrophils alone had only a small effect on

8 the cytosolic $\left[\mathrm{Ca}^{2+}\right]$ (Fig. 2a and b). However, when given together, endothelial

9 cytosolic $\mathrm{Ca}^{2+}$ concentration strongly increased (Fig. 2a and b). Leukocyte-dependent

10 increases in endothelial $\left[\mathrm{Ca}^{2+}\right]_{\mathrm{i}}$ were rarely seen during rolling or initial arrest of

11 leukocytes but during crawling and also during the transmigration phase (Extended

12 Data Fig. 3a). After knock-down of endothelial PIEZO1, calcium transients induced by

13 leukocytes in the presence of low flow were strongly reduced or absent (Fig. 2c and 14 d).

We then tested the potential involvement of PIEZO1 in the induction of 16 downstream signaling events mediating leukocyte-induced opening of endothelial 17 junctions. Again, low flow alone or addition of PMNs had hardly any effect on the phosphorylation of PYK2, SRC and the myosin light chain (MLC) in endothelial cells. However, application of both flow and PMNs synergistically induced endothelial PYK2, SRC and MLC phosphorylation, and this effect was strongly reduced after

21 knock-down of PIEZO1 (Fig. 2e,f). The effect of PMNs and flow was mimicked by 22 application of Yoda1, and this effect was blocked after knock-down of PIEZO1 (Fig. $232 \mathrm{~g}, \mathrm{~h})$. Inhibition of endothelial PYK2 or SRC by PF431396 or PP2, respectively, 24 reduced basal transmigration and blocked Yoda1-induced increases in PMN 25 transmigration (Fig. 2i). These data strongly indicate that PMNs and low flow 26 synergistically induce downstream signaling events through endothelial PIEZO1 
1 resulting in the opening of endothelial junctions and leukocyte transmigration.

2 Consistent with this, we also observed synergism in the ability of flow and PMNs to

3 induce internalization of VE-cadherin, an effect strongly inhibited after siRNA-

4 mediated suppression of PIEZO1 expression (Fig. 2j).

5

\section{Endothelial PIEZO1 is activated by flow-induced ICAM-1 clustering}

Since engagement of endothelial ICAM-1 by leukocyte $\beta 2$ integrins is essential for induction of increases in $\left[\mathrm{Ca}^{2+}\right]_{i}$ and diapedesis, we suppressed expression of endothelial ICAM-1 and found that this strongly inhibited PMN-induced $\mathrm{Ca}^{2+}$ transients as well as PYK2, SRC and MLC phosphorylation (Extended Fig. 3b-e). Clustering of ICAM-1 using beads coated with anti-ICAM-1 antibodies mimicked the effect of PMNs and induced $\mathrm{Ca}^{2+}$ transients as well as phosphorylation of PYK2, SRC and MLC synergistically with low flow (Fig. 3a,b, Extended Data Fig. 3f,g). The effects of ICAM-1 clustering were inhibited after knock-down of PIEZO1 and ICAM-1 (Fig. 3a-d). To test the direct effect of ICAM-1 clustering on PIEZO1-dependent signaling, we cross-linked bound anti-ICAM-1 antibodies. As shown in Fig. 3e-h, clustering of ICAM-1 induced by antibody cross-linking mimicked the effect of PMNs and of anti-ICAM-1 beads and induced $\mathrm{Ca}^{2+}$ transients as well as phosphorylation of PYK2, SRC and MLC synergistically with application of low flow in a PIEZO1dependent manner. This strongly indicates that clustering and activation of ICAM-1 by leukocytes in the presence of flow results in PIEZO1-mediated downstream signaling leading to the opening of endothelial junctions.

\section{ICAM-1 clustering and flow synergistically increase membrane tension}

To analyze how ICAM-1 clustering leads to PIEZO1 activation, we determined membrane tension using the fluorescent lipid tension sensor, FliptR. We found that 
1 clustering of ICAM-1 leads to a small increase in endothelial membrane tension (Fig.

$24 a$ and b). Low flow, which by itself had no significant effect on endothelial membrane

3 tension, when given together with ICAM-1 clustering agents, resulted in a very strong

4 increase in plasma membrane tension (Fig. $4 a$ and b). This indicates that low flow

5 and ICAM-1 clustering synergistically increase endothelial membrane tension. Since

6 ICAM-1 clustering has been shown to induce localized actin polymerization and

7 myosin activity resulting in a localized reorganization of the cortical cytoskeleton ${ }^{25}$,

8 we analyzed the effect of cytochalasin $D$ and blebbistatin on membrane tension and

9 on phosphorylation of PYK2, SRC and MLC induced by ICAM-1 clustering. Both

10 agents blocked ICAM-1-dependent changes in membrane tension and downstream

11 signalling (Fig. 4c,d, Extended Data Fig. 4a,b). We then tested whether increased

12 membrane tension and downstream signaling induced by ICAM-1 clustering involves

13 the actin adapter proteins $\alpha$-actinin-4 and cortactin which have been shown to be

14 recruited after clustering of ICAM-1 and to be required for ICAM-1-mediated

15 endothelial actin filament branching as well as for ICAM-1-dependent 16 transendothelial migration of neutrophils ${ }^{26-28}$. As shown in Fig. 4e,f and Extended 17 Data Fig. 4c-e, siRNA-mediated knock-down of the RNAs encoding $\alpha$-actinin-4 and cortactin (ACTN4 and CTTN, respectively) blocked the effect of ICAM-1 clustering on membrane tension and downstream signaling.

\section{Discussion}

We here report that the mechanosensitive cation channel PIEZO1 plays a critical role in transendothelial migration of leukocytes in vitro and in vivo by integrating

24 coincident mechanical signals induced by low levels of fluid shear stress and by 25 leukocyte-dependent clustering of ICAM-1. PIEZO1 thereby mediates an increase in $26\left[\mathrm{Ca}^{2+}\right]_{\mathrm{i}}$ which leads to localized opening of the endothelial barrier (Fig. $4 \mathrm{~g}$ ). Recent 
1 data reported that TRPC6 is critically involved in leukocyte-induced increases in

2 endothelial $\left[\mathrm{Ca}^{2+}\right]_{i}$ during leukocyte transendothelial migration ${ }^{17}$. In in vitro

3 experiments, we were not able to observe a contribution of TRPC6 in leukocyte-

4 induced calcium transients, but it could well be that both PIEZO1 and TRPC6 operate

5 in parallel under in vivo conditions or that PIEZO1 is involved in the initiation of

6 leukocyte extravasation whereas TRPC6 mediates increases in $\left[\mathrm{Ca}^{2+}\right]_{\mathrm{i}}$ mainly at later

7 stages of diapedesis. However, our study considerably differed from the study reported by Weber et al. in that we investigated the role of PIEZO1 in the presence of physiological flow conditions and therefore also addressed whether the local hemodynamic environment of the adhering and transmigrating leukocyte has an

11 effect on leukocyte-induced downstream signaling and transmigration.

ICAM-1 is a central endothelial adhesion receptor that functions as a ligand for ß2 integrins on leukocytes and promotes leukocyte spreading, migration and transmigration $^{29,30}$. Engagement of ICAM-1 leads to clustering of ICAM-1 molecules and cytoskeletal changes such as actin polymerization, MLC phosphorylation and actomyosin contractility, which promote junctional opening ${ }^{16,25,30,31}$. ICAM-1 also promotes increase in $\left[\mathrm{Ca}^{2+}\right]_{i}$ levels ${ }^{12,32}$, which has been shown to lead to activation of SRC via protein kinase $\mathrm{C}^{14}$, and ICAM-1-mediated activation of SRC and PYK2 has been shown to be required for VE-cadherin-dependent leukocyte transendothelial migration $^{33}$. This involves direct phosphorylation of VE-cadherin ${ }^{33-35}$ as well as

21 indirect regulation of VE-cadherin through VE-PTP ${ }^{36}$ or by phosphorylation of $\beta$ 22 catenin $^{37}$. How ICAM-1 clustering induces activation of these downstream signaling events resulting in junctional opening and transendothelial migration was unclear. Our data indicate that downstream signaling through ICAM-1 requires co-activation of PIEZO1 by fluid shear stress and ICAM-1-induced reorganization of the cortical cytoskleleton. 
Various mechanical stimuli acting on cellular membranes have been shown to

2 be able to activate PIEZO1. These include exposure to fluid shear stress, mechanical

3 indentation of the cell surface, compression of the cell membrane or forces generated

4 at the cell-cell or cell-matrix interface ${ }^{19,38}$. Our data show that low-level fluid shear

5 stress as well as interaction of leukocytes with the endothelial surface act in an

6 synergistic manner to activate endothelial PIEZO1 and to initiate leukocyte

7 transendothelial migration. In postcapillary venules, the place where leukocyte extravasation mainly takes place, the shear stress exerted by the flowing blood is

9 relatively low at about $1-2$ dynes $/ \mathrm{cm}^{2}{ }^{39,40}$, a shear rate hardly able to induce PIEZO1 mediated signaling ${ }^{24}$. Consistent with this, we saw only very small increases in $\left[\mathrm{Ca}^{2+}\right]_{\mathrm{i}}$

11 and no significant increase in the phosphorylation of PYK2, SRC or MLC in response

12 to fluid shear stress of 1.2 dynes $/ \mathrm{cm}^{2}$. Similarly, when ICAM-1 clustering was induced

13 in TNFa-pretreated endothelial cells by PMNs or anti-ICAM-1 antibodies, only small

14 increases in $\left[\mathrm{Ca}^{2+}\right]_{\mathrm{i}}$ and phosphorylation of PYK2, SRC and MLC could be observed,

15 which were further reduced after suppression of PIEZO1 expression. However, when

16 endothelial ICAM-1 clustering was induced while exposing cells to low flow, 17 downstream signaling was strongly activated in a PIEZO1-dependent manner. This raised the question as to how ICAM-1 clustering promotes PIEZO1 activation. Both ICAM-1 clustering and adhesion of leukocytes to endothelial cells have been shown to induce stiffening of the endothelial surface and to induce traction stress ${ }^{25,41-44}$.

21 These endothelial responses are due to increased actin polymerization and 22 actomyosin contractility of the cortical cytoskeleton which lead to increased cortical 23 tension $^{45,46}$ and require recruitment of the actin adapter proteins $\alpha$-actinin- 4 and 24 cortactin $^{25,26}$. Since the plasma membrane and the underlying cortical cytoskeleton 25 are closely interconnected ${ }^{45,46}$, changes in the actomyosin cortical tension directly 26 affect plasma membrane tension ${ }^{46}$ and therefore are likely to regulate PIEZO1 
1 activity. Consistent with this, we found that inhibition of actin polymerization and

2 myosin activity as well as siRNA-mediated knock-down of $\alpha$-actinin-4 and cortactin

3 blocked ICAM-1-mediated increases in membrane tension as well as PIEZO1-

4 dependent downstream signaling required for leukocyte transendothelial migration.

$5 \quad$ Recent data indicate that changes in plasma membrane tension are restricted

6 to subcellular domains of endothelial cells as local increases in membrane tension

7 lead only to local activation of mechanosensitive ion channels such as PIEZO ${ }^{47}$.

8 The finding that leukocyte-induced endothelial downstream signaling and diapedesis

9 require PIEZO1 and flow is consistent with earlier observations, which showed that

10 fluid shear stress promotes transendothelial leukocyte migration ${ }^{48-50}$. Our data

11 identify a novel synergism of local hemodynamic forces and initial endothelial

12 leukocyte adhesion to induce plasma membrane tension and endothelial signaling 13 events which promote leukocyte extravasation. The discovery of a novel 14 mechanosensing and mechanosignalling process required for the initial phase of 15 leukocyte diapedesis may also lead to new anti-inflammatory therapeutic approaches. 


\section{Acknowledgements}

2 The authors wish to thank Svea Hümmer for secretarial help, Yin Hao (Instrument

3 Analysis Center of Xi'an Jiaotong University) for assistance with fluorescence-lifetime

4 imaging microscopy and Shuya Liu, Martina Finkbeiner, Ulrike Krüger and Claudia

5 Ullmann for technical help. This work was supported by the Collaborative Research

6 Centre 834 of the German Research Foundation (S.O.), the Collaborative Research

7 Center 1348 of the German Research Foundation (D.V.) and the National Natural

8 Science Foundation of China (grant \#81870220, S.P.W), Shaanxi Natural Science

9 Fund for Distinguished Young Scholars of China (S2020-JC-JQ-0239, S.P.W).

\section{Author contributions}

S.P.W. initiated and designed the study, performed experiments, analyzed data and 13 wrote the manuscript; Y.S. performed in vitro experiments; T.M. and R.I.S. performed 14 in vivo experiments; B.S. helped with in vitro and in vivo experiments; L.X. and Z.Y. 15 helped with in vitro experiments; N.W. supervised part of the study and discussed data; D.V. supervised part of the in vivo experiments and analyzed and discussed data; S.O. designed and supervised the study, discussed data and wrote the manuscript. All authors commented on the manuscript.

\section{Author information}

21 The authors declare no competing financial interests. Correspondence and request

22 for materials should be addressed to S.O. (stefan.offermanns@mpi-bn.mpg.de) or 23 S.P.W. (shengpeng.wang@xjtu.edu.cn). 


\section{References}

1 Springer, T. A. Traffic signals for lymphocyte recirculation and leukocyte emigration: the multistep paradigm. Cell 76, 301-314, doi:10.1016/00928674(94)90337-9 (1994).

2 Goswami, D. \& Vestweber, D. How leukocytes trigger opening and sealing of gaps in the endothelial barrier. F1000Res 5, doi:10.12688/f1000research.9185.1 (2016).

3 Alon, R. \& van Buul, J. D. Leukocyte Breaching of Endothelial Barriers: The Actin Link. Trends Immunol 38, 606-615, doi:10.1016/j.it.2017.05.002 (2017).

4 Butcher, E. C. Leukocyte-endothelial cell recognition: three (or more) steps to specificity and diversity. Cell 67, 1033-1036, doi:10.1016/0092-8674(91)90279-8 (1991).

5 Ley, K., Laudanna, C., Cybulsky, M. I. \& Nourshargh, S. Getting to the site of inflammation: the leukocyte adhesion cascade updated. Nat Rev Immunol 7, 678-689, doi:10.1038/nri2156 (2007).

6 Hordijk, P. L. Recent insights into endothelial control of leukocyte extravasation. Cell Mol Life Sci 73, 1591-1608, doi:10.1007/s00018-016-2136-y (2016).

7 Gerhardt, T. \& Ley, K. Monocyte trafficking across the vessel wall. Cardiovasc Res 107, 321-330, doi:10.1093/cvr/cvv147 (2015).

8 Vestweber, D. How leukocytes cross the vascular endothelium. Nat Rev Immunol 15, 692-704, doi:10.1038/nri3908 (2015).

9 Nourshargh, S. \& Alon, R. Leukocyte migration into inflamed tissues. Immunity 41, 694-707, doi:10.1016/j.immuni.2014.10.008 (2014).

10 Muller, W. A. Transendothelial migration: unifying principles from the endothelial perspective. Immunol Rev 273, 61-75, doi:10.1111/imr.12443 (2016).

11 Schimmel, L., Heemskerk, N. \& van Buul, J. D. Leukocyte transendothelial migration: A local affair. Small GTPases 8, 1-15, doi:10.1080/21541248.2016.1197872 (2017).

12 Huang, A. J. et al. Endothelial cell cytosolic free calcium regulates neutrophil migration across monolayers of endothelial cells. J Cell Biol 120, 1371-1380, doi:10.1083/jcb.120.6.1371 (1993).

13 Su, W. H., Chen, H. I., Huang, J. P. \& Jen, C. J. Endothelial $[\mathrm{Ca}(2+)](\mathrm{i})$ signaling during transmigration of polymorphonuclear leukocytes. Blood 96, 3816-3822 (2000).

14 Etienne-Manneville, S. et al. ICAM-1-coupled cytoskeletal rearrangements and transendothelial lymphocyte migration involve intracellular calcium signaling in brain endothelial cell lines. J Immunol 165, 3375-3383 (2000).

15 Kielbassa-Schnepp, K. et al. Endothelial intracellular Ca2+ release following monocyte adhesion is required for the transendothelial migration of monocytes. Cell Calcium 30, 29-40, doi:10.1054/ceca.2001.0210 (2001).

16 Dalal, P. J. et al. Spatiotemporal restriction of endothelial cell calcium signaling is required during leukocyte transmigration. $J$ Exp Med 218, doi:10.1084/jem.20192378 (2021). 
17 Weber, E. W. et al. TRPC6 is the endothelial calcium channel that regulates leukocyte transendothelial migration during the inflammatory response. J Exp Med 212, 1883-1899, doi:10.1084/jem.20150353 (2015).

18 Coste, B. et al. Piezo proteins are pore-forming subunits of mechanically activated channels. Nature 483, 176-181, doi:10.1038/nature10812 (2012).

19 Murthy, S. E., Dubin, A. E. \& Patapoutian, A. Piezos thrive under pressure: mechanically activated ion channels in health and disease. Nature reviews. Molecular cell biology 18, 771-783, doi:10.1038/nrm.2017.92 (2017).

20 Zhao, Q. et al. Structure and mechanogating mechanism of the Piezo1 channel. Nature 554, 487-492, doi:10.1038/nature25743 (2018).

21 Lewis, A. H. \& Grandl, J. Mechanical sensitivity of Piezo1 ion channels can be tuned by cellular membrane tension. Elife 4, doi:10.7554/eLife.12088 (2015).

22 Syeda, R. et al. Piezo1 Channels Are Inherently Mechanosensitive. Cell Rep 17, 1739-1746, doi:10.1016/j.celrep.2016.10.033 (2016).

23 Ranade, S. S. et al. Piezo1, a mechanically activated ion channel, is required for vascular development in mice. Proc Natl Acad Sci U S A 111, 10347-10352, doi:10.1073/pnas.1409233111 (2014).

$24 \mathrm{Li}$, J. et al. Piezo1 integration of vascular architecture with physiological force. Nature 515, 279-282, doi:10.1038/nature13701 (2014).

25 Lessey-Morillon, E. C. et al. The RhoA guanine nucleotide exchange factor, LARG, mediates ICAM-1-dependent mechanotransduction in endothelial cells to stimulate transendothelial migration. J Immunol 192, 3390-3398, doi:10.4049/jimmunol.1302525 (2014).

26 Schaefer, A. et al. Actin-binding proteins differentially regulate endothelial cell stiffness, ICAM-1 function and neutrophil transmigration. J Cell Sci 127, 44704482, doi:10.1242/jcs.154708 (2014).

27 Schnoor, M. et al. Cortactin deficiency is associated with reduced neutrophil recruitment but increased vascular permeability in vivo. J Exp Med 208, 17211735, doi:10.1084/jem.20101920 (2011).

28 Celli, L., Ryckewaert, J. J., Delachanal, E. \& Duperray, A. Evidence of a functional role for interaction between ICAM-1 and nonmuscle alpha-actinins in leukocyte diapedesis. J Immunol 177, 4113-4121, doi:10.4049/jimmunol.177.6.4113 (2006).

29 Lawson, C. \& Wolf, S. ICAM-1 signaling in endothelial cells. Pharmacol Rep 61, 22-32, doi:10.1016/s1734-1140(09)70004-0 (2009).

30 van Buul, J. D., Kanters, E. \& Hordijk, P. L. Endothelial signaling by Ig-like cell adhesion molecules. Arterioscler Thromb Vasc Biol 27, 1870-1876, doi:10.1161/ATVBAHA.107.145821 (2007).

31 Wee, H., Oh, H. M., Jo, J. H. \& Jun, C. D. ICAM-1/LFA-1 interaction contributes to the induction of endothelial cell-cell separation: implication for enhanced leukocyte diapedesis. Exp Mol Med 41, 341-348, doi:10.3858/emm.2009.41.5.038 (2009).

32 Clayton, A. et al. Cellular activation through the ligation of intercellular adhesion molecule-1. J Cell Sci 111 ( Pt 4), 443-453 (1998). 
33 Allingham, M. J., van Buul, J. D. \& Burridge, K. ICAM-1-mediated, Src- and Pyk2dependent vascular endothelial cadherin tyrosine phosphorylation is required for leukocyte transendothelial migration. $J$ Immunol 179, 4053-4064, doi:10.4049/jimmunol.179.6.4053 (2007).

34 Alcaide, P. et al. p120-Catenin prevents neutrophil transmigration independently of RhoA inhibition by impairing Src dependent VE-cadherin phosphorylation. Am J Physiol Cell Physiol 303, C385-395, doi:10.1152/ajpcell.00126.2012 (2012).

35 Wallez, Y. et al. Src kinase phosphorylates vascular endothelial-cadherin in response to vascular endothelial growth factor: identification of tyrosine 685 as the unique target site. Oncogene 26, 1067-1077, doi:10.1038/sj.onc.1209855 (2007).

36 Soni, D. et al. Pyk2 phosphorylation of VE-PTP downstream of STIM1-induced $\mathrm{Ca}(2+)$ entry regulates disassembly of adherens junctions. Am J Physiol Lung Cell Mol Physiol 312, L1003-L1017, doi:10.1152/ajplung.00008.2017 (2017).

37 van Buul, J. D., Anthony, E. C., Fernandez-Borja, M., Burridge, K. \& Hordijk, P. L. Proline-rich tyrosine kinase 2 (Pyk2) mediates vascular endothelial-cadherinbased cell-cell adhesion by regulating beta-catenin tyrosine phosphorylation. $J$ Biol Chem 280, 21129-21136, doi:10.1074/jbc.M500898200 (2005).

$38 \mathrm{Wu}$, J., Lewis, A. H. \& Grandl, J. Touch, Tension, and Transduction - The Function and Regulation of Piezo Ion Channels. Trends Biochem Sci 42, 57-71, doi:10.1016/j.tibs.2016.09.004 (2017).

39 Zhao, R. et al. Rescue of embryonic lethality in reduced folate carrier-deficient mice by maternal folic acid supplementation reveals early neonatal failure of hematopoietic organs. J Biol Chem 276, 10224-10228 (2001).

40 Morikis, V. A. \& Simon, S. I. Neutrophil Mechanosignaling Promotes Integrin Engagement With Endothelial Cells and Motility Within Inflamed Vessels. Front Immunol 9, 2774, doi:10.3389/fimmu.2018.02774 (2018).

41 Yeh, Y. T. et al. Three-dimensional forces exerted by leukocytes and vascular endothelial cells dynamically facilitate diapedesis. Proc Natl Acad Sci U S A 115, 133-138, doi:10.1073/pnas.1717489115 (2018).

42 Wang, Q. \& Doerschuk, C. M. Neutrophil-induced changes in the biomechanical properties of endothelial cells: roles of ICAM-1 and reactive oxygen species. $J$ Immunol 164, 6487-6494, doi:10.4049/jimmunol.164.12.6487 (2000).

43 Wang, Q. et al. Changes in the biomechanical properties of neutrophils and endothelial cells during adhesion. Blood 97, 660-668, doi:10.1182/blood.v97.3.660 (2001).

44 Liu, Z., Sniadecki, N. J. \& Chen, C. S. Mechanical Forces in Endothelial Cells during Firm Adhesion and Early Transmigration of Human Monocytes. Cell Mol Bioeng 3, 50-59, doi:10.1007/s12195-010-0105-3 (2010).

45 Kelkar, M., Bohec, P. \& Charras, G. Mechanics of the cellular actin cortex: From signalling to shape change. Curr Opin Cell Biol 66, 69-78, doi:10.1016/j.ceb.2020.05.008 (2020).

46 Sitarska, E. \& Diz-Munoz, A. Pay attention to membrane tension: Mechanobiology of the cell surface. Curr Opin Cell Biol 66, 11-18, doi:10.1016/j.ceb.2020.04.001 (2020). 
47 Shi, Z., Graber, Z. T., Baumgart, T., Stone, H. A. \& Cohen, A. E. Cell Membranes Resist Flow. Cell 175, 1769-1779 e1713, doi:10.1016/j.cell.2018.09.054 (2018).

48 Cinamon, G., Shinder, V. \& Alon, R. Shear forces promote lymphocyte migration across vascular endothelium bearing apical chemokines. Nat Immunol 2, 515522, doi:10.1038/88710 (2001).

49 Cuvelier, S. L. \& Patel, K. D. Shear-dependent eosinophil transmigration on interleukin 4-stimulated endothelial cells: a role for endothelium-associated eotaxin-3. J Exp Med 194, 1699-1709, doi:10.1084/jem.194.12.1699 (2001).

50 Kitayama, J., Hidemura, A., Saito, H. \& Nagawa, H. Shear stress affects migration behavior of polymorphonuclear cells arrested on endothelium. Cell Immunol 203, 39-46, doi:10.1006/cimm.2000.1671 (2000). 
Figures and figure legends

2

a

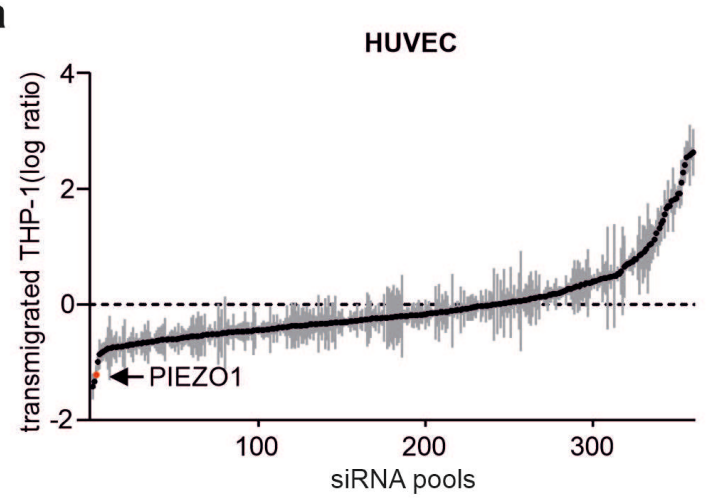

b C

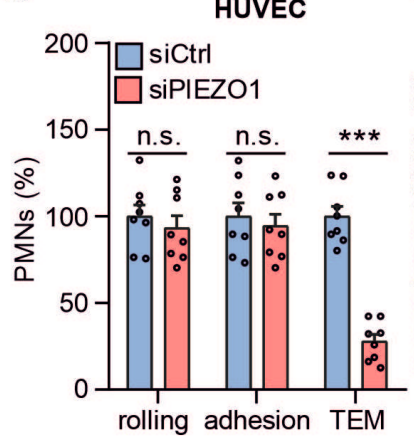

MLEC

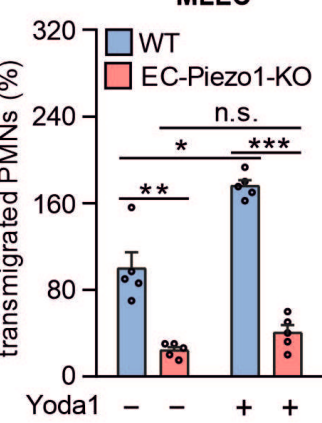

d

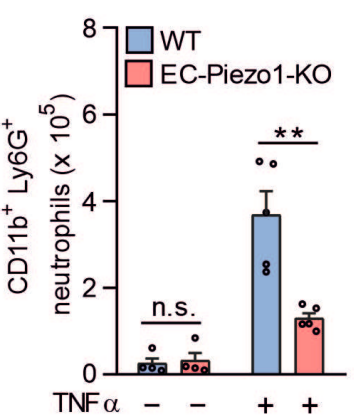

e

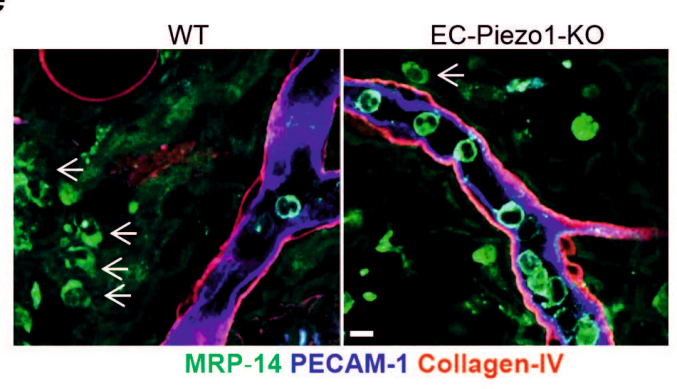

f

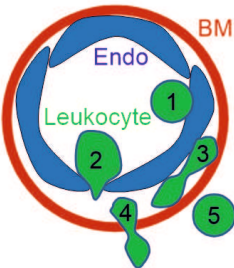

g

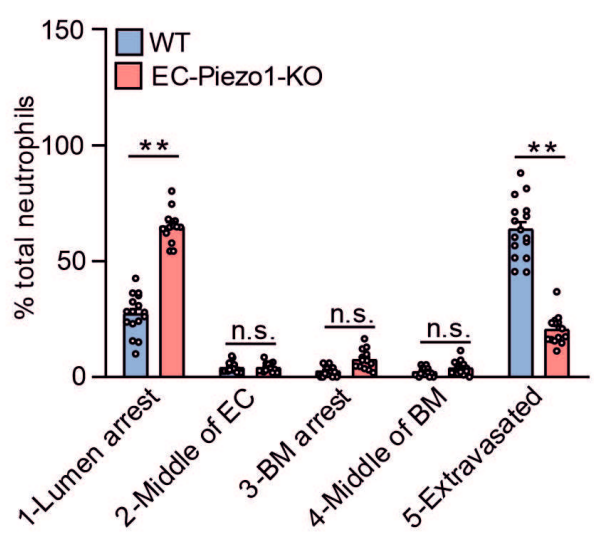

h

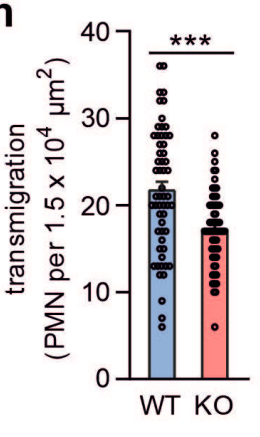

i

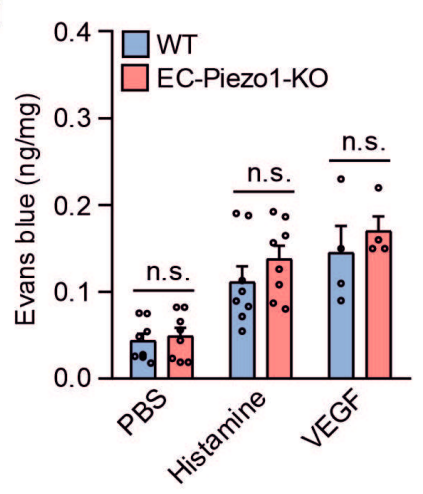

Figure 1. PIEZO1 mediates leukocyte transendothelial migration in vitro and in vivo.

(a) HUVECs pretreated with $10 \mathrm{ng} / \mathrm{ml}$ TNFa were transfected with 360 siRNAs pools against RNAs encoding transmembrane proteins expressed in endothelial cells and were then exposed to THP-1 monocytic cells for 3 hours. Shown is the ratio of THP-1 cells which transmigrated the HUVEC monolayer transfected with a particular siRNA pool and with control siRNA. The plot shows the ranked average ratios of three independent experiments. (b) HUVECs were transfected with control (siCtrl) or PIEZO1-specific siRNA (siPIEZO1), and rolling, adhesion and transmigration of human PMNs applied together with flow $\left(1.2\right.$ dynes $\left./ \mathrm{cm}^{2}\right)$ were analyzed $(n=8$ per group). Cells treated with control siRNA were set as $100 \%$. (c) Mouse lung 
1 endothelial cells (MLECs) were isolated from wild-type (WT) and EC-Piezo1-KO mice

2 and transmigration of mouse PMNs was determined after pretreament without or with

$31 \mu \mathrm{M}$ Yoda1 for $15 \mathrm{~min}(\mathrm{n}=5)$. (d) Wild-type (WT) and endothelium-specific PIEZO1

4 deficient mice (EC-Piezo1-KO) were injected intraperitoneally with PBS or $500 \mathrm{ng}$ of

$5 \mathrm{TNFa}$, and the number of peritoneal $\mathrm{CD} 11 \mathrm{~b}^{+} ; \mathrm{Ly} 6 \mathrm{G}^{+}$neutrophils was determined by

6 flow cytometry ( $n=4$ mice (-TNF $\alpha)$; $n=5$ mice (+TNFa)). (e-g) Wild-type (WT) and EC-

7 Piezo1-KO mice were treated with croton oil on one ear. $6 \mathrm{~h}$ later, animals were killed

8 and ears were immunostained as whole mounts with antibodies against PECAM-1

9 (blue, endothelium), collagen-IV (red, basement membrane) and MRP14 (green,

10 neutrophil). Arrows indicate neutrophils. Scale bar: $10 \mu \mathrm{m}$. (e) Representative images

11 of stained ears. (f) Schematic drawing illustrating the criteria to delineate the 5 12 positions in which leukocyte are found during extravasation. (g) Distribution pattern of 13 neutrophil positions relative to the endothelium and basement membrane $(n=16$ mice 14 (WT); n=14 mice (EC-Piezo1-KO), 3-5 vessels were analyzed per animal). (h) WT 15 and EC-Piezo1-KO mice were analyzed by intravital microscopy of cremaster 16 venules 4 hours after injection of $50 \mathrm{ng} \mathrm{IL-1 \beta}$ for extravasated leukocytes ( $\mathrm{n}=9$ mice per group; 4-10 measurements per animal). (i) Evans blue extravasation was assessed after subcutaneous injection of $20 \mu \mathrm{l}$ of PBS without or with $100 \mu \mathrm{M}$ of histamine or $100 \mathrm{ng} / \mathrm{ml}$ of VEGF ( $\mathrm{n}=8$ mice (PBS and histamine); $\mathrm{n}=4$ mice (VEGF)).

20 Shown are mean values \pm s.e.m.; ${ }^{*} P \leq 0.05$; ${ }^{* *} P \leq 0.01$; ${ }^{* * *} P \leq 0.001$ (unpaired two21 tailed $t$-test). 

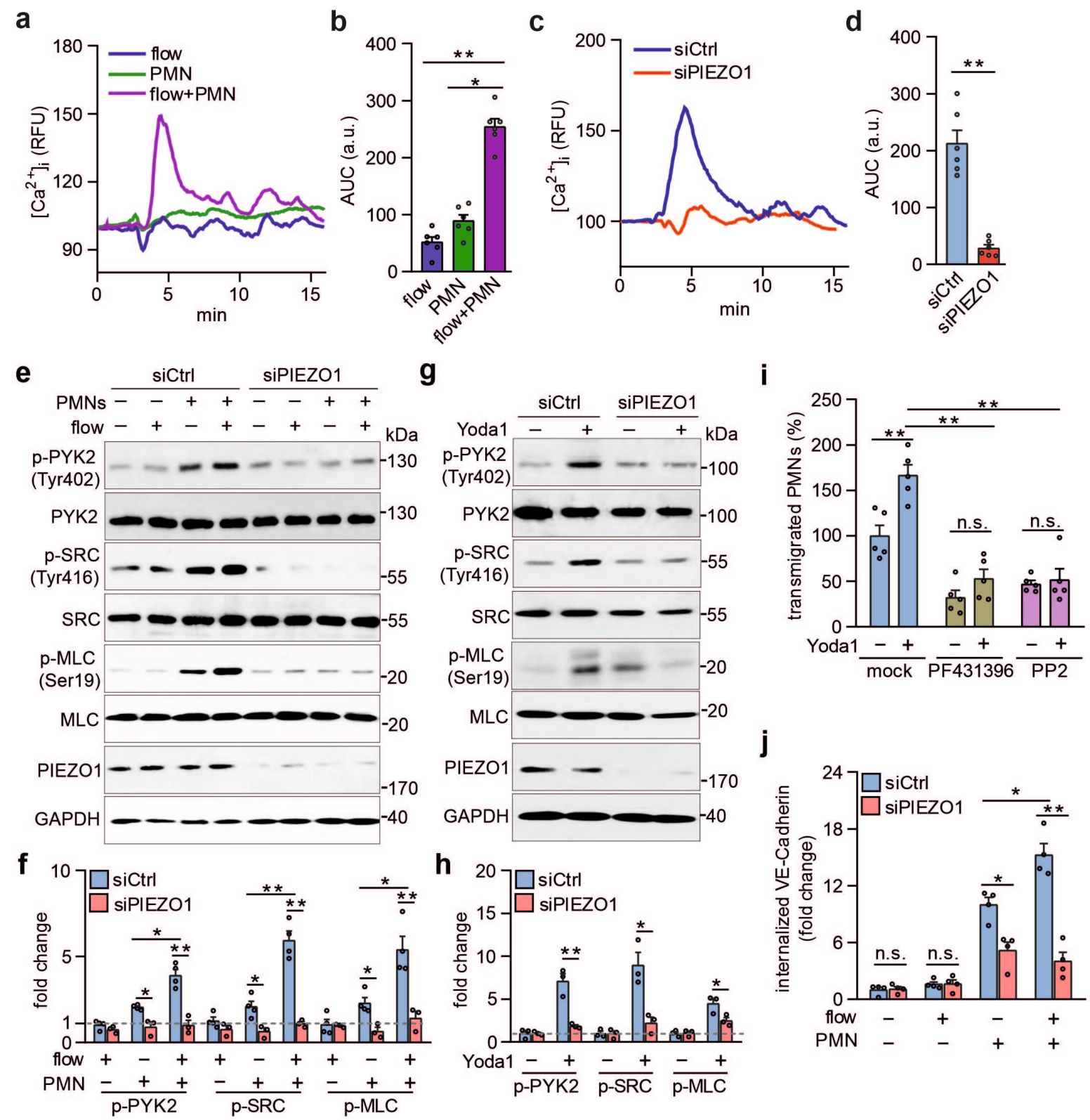

2 Figure 2. Leukocytes and flow synergistically induce PIEZO1 activation to stimulate 3 endothelial downstream signaling.

4 (a-d) Untransfected HUVECs (a,b) or HUVECs transfected with control (siCtrl) or 5 PIEZO1-specific siRNA (siPIEZO1) $(\mathrm{c}, \mathrm{d})$ were preactivated with TNFa, loaded with 6 Fluo-4 and were then exposed to PMNs alone, to low flow $\left(1.2 \mathrm{dynes} / \mathrm{cm}^{2}\right)$ alone or 7 to both $(a, b)$ or to PMNs and low flow together $(c, d) \cdot\left[\mathrm{Ca}^{2+}\right]_{i}$ was determined as 8 fluorescence intensity (RFU, relative fluorescence units) (a, c). b and d show the area 9 under curve (AUC) of the $\left[\mathrm{Ca}^{2+}\right]_{\mathrm{i}}$-trace from 6 independent experiments (a.u., 10 arbitrary units). (e-h) Immunoblot analysis of total and phosphorylated PYK2, SRC 11 and MLC in lysates of TNFa-activated HUVECs transfected with control siRNA (siCtrl) 12 or siRNA directed against PIEZO1 and incubated without or with human PMNs in the 
1 absence or presence of low flow $\left(1.2\right.$ dynes $\left./ \mathrm{cm}^{2}\right)$ (e) or without or with $5 \mu \mathrm{M}$ Yoda1

2 (g). Immunoblot analysis of PIEZO1 and GAPDH served as controls. Bar diagrams

3 (f,h) show the densitometric analysis of 3 independent experiments. (i)

4 Transmigration of human PMNs across TNFa-activated HUVECs preincubated for 30

5 min with the PYK2 and SRC inhibitors PF431396 (10 $\mu \mathrm{M})$ and PP2 (10 $\mu \mathrm{M})$,

6 respectively ( $n=5$ independent experiments). (j) HUVECs transfected with control

7 (siCtrl) or PIEZO1-specific siRNA (siPIEZO1) were preactivated with TNFa and were

8 then exposed to PMNs alone, to low flow $\left(1.2\right.$ dynes $\left./ \mathrm{cm}^{2}\right)$ alone or to both. After 15

9 minutes VE-cadherin internalization was determined as described in the Methods

10 ( $n=4$ ). Shown are mean values \pm s.e.m.; ${ }^{*} P \leq 0.05$; ${ }^{* *} P \leq 0.01$ (unpaired two-tailed $t$ 11 test). 
a

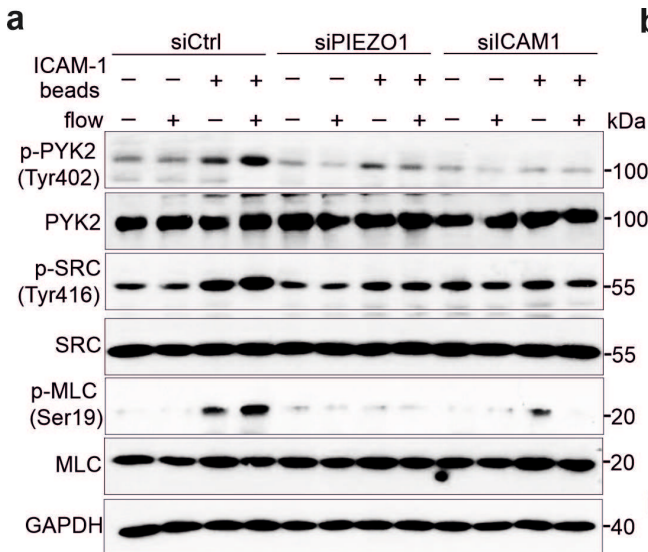

e

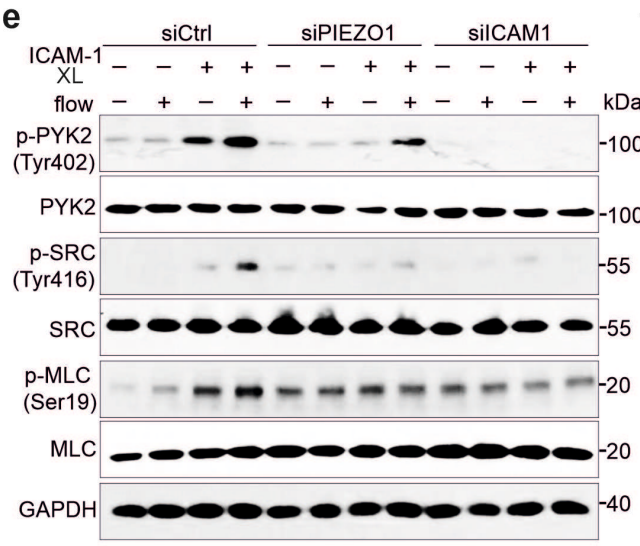

b

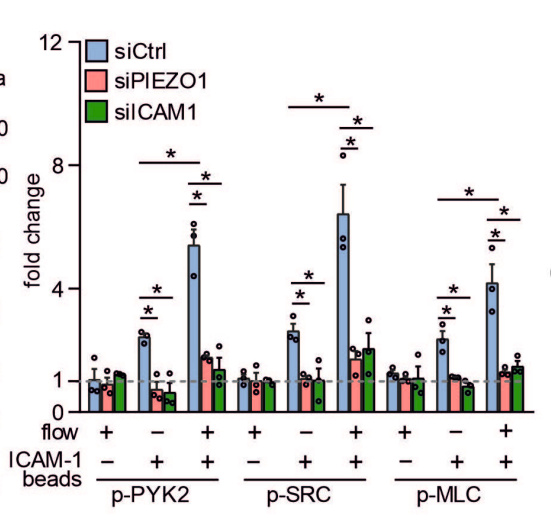

C

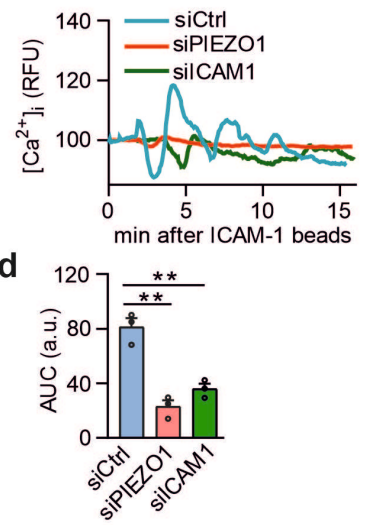

g

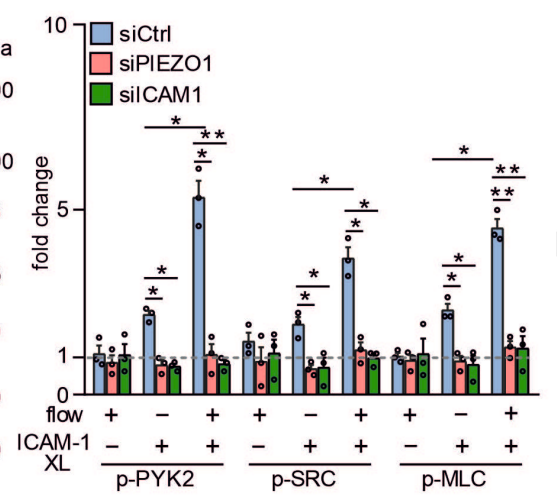

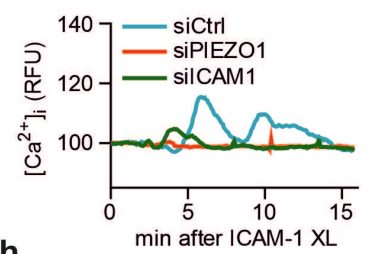

h

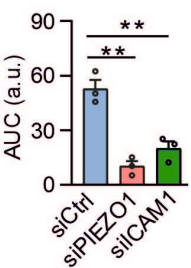

Figure 3. Endothelial PIEZO1 activation by leukocytes involves ICAM-1 activation and flow.

4 (a-h) TNFa-activated HUVECs transfected with control siRNA (siCtrl) or siRNA

5 directed against ICAM-1 or PIEZO1 were exposed to low flow alone, anti-ICAM-1

6 antibody beads (ICAM-1 beads) alone or both (a-d) or to low flow alone, anti-ICAM-1

7 clustering antibodies (ICAM-1 XL) or both (e-h), and immunoblot analysis of total and

8 phosphorylated PYK2, SRC and MLC was performed. Immunoblot analysis of

9 GAPDH served as controls. Bar diagrams (b,f) show the densitometric analysis of 3

10 independent experiments. Alternatively, the free $\left[\mathrm{Ca}^{2+}\right]_{\mathrm{i}}$ was determined after loading

11 of cells with Fluo4 (c,g). Bar diagrams (d,h) show the area under the curve (AUC) of

12 the $\left[\mathrm{Ca}^{2+}\right]_{i}$-trace from 3 independent experiments (a.u., arbitrary units). Shown are

13 mean values \pm s.e.m.; ${ }^{*} \mathrm{P} \leq 0.05$; ${ }^{* *} \mathrm{P} \leq 0.01$ (unpaired two-tailed $t$-test). 
a
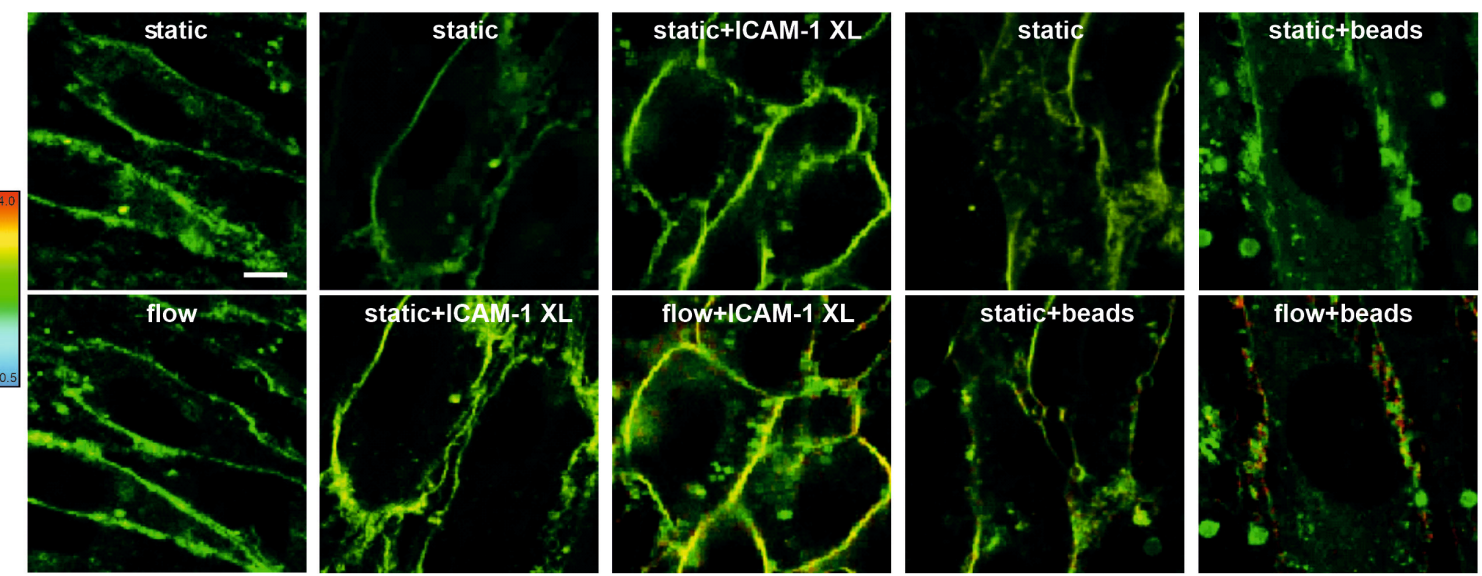

b

C

d
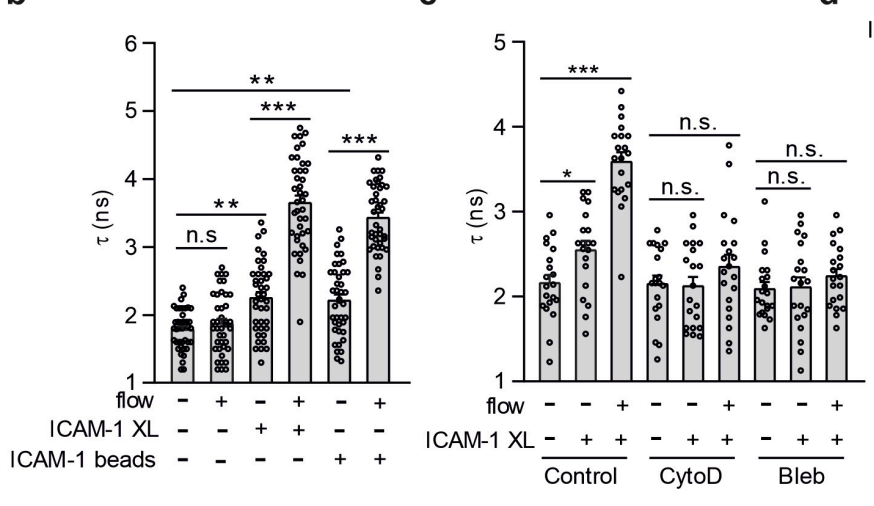

ICAM-1XL $\frac{\text { Control }}{--++} \frac{\text { Cytochalasin D }}{--++} \frac{\text { Blebbistatin }}{-+++}$

ICAM- 1 beads

e

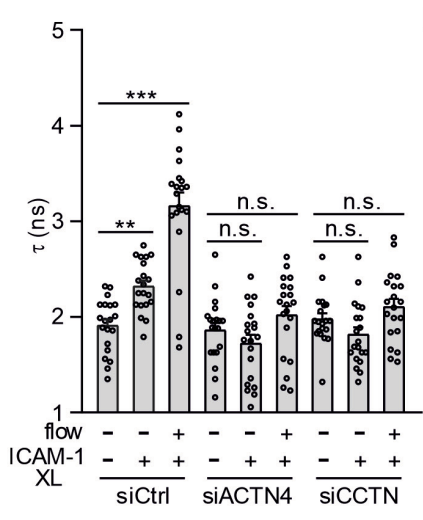

f

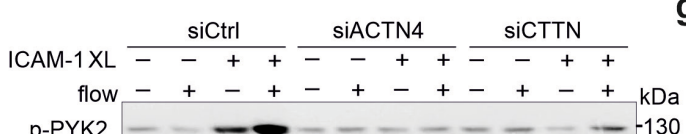
P-PYK2
(Tyr402)
PYK2

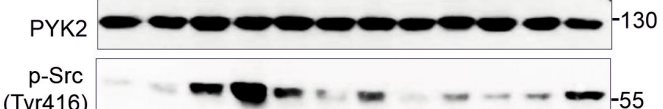
$\begin{gathered}\text { (Tyr416) } \\ \text { T-Src }\end{gathered}-\infty-\cdots--5$
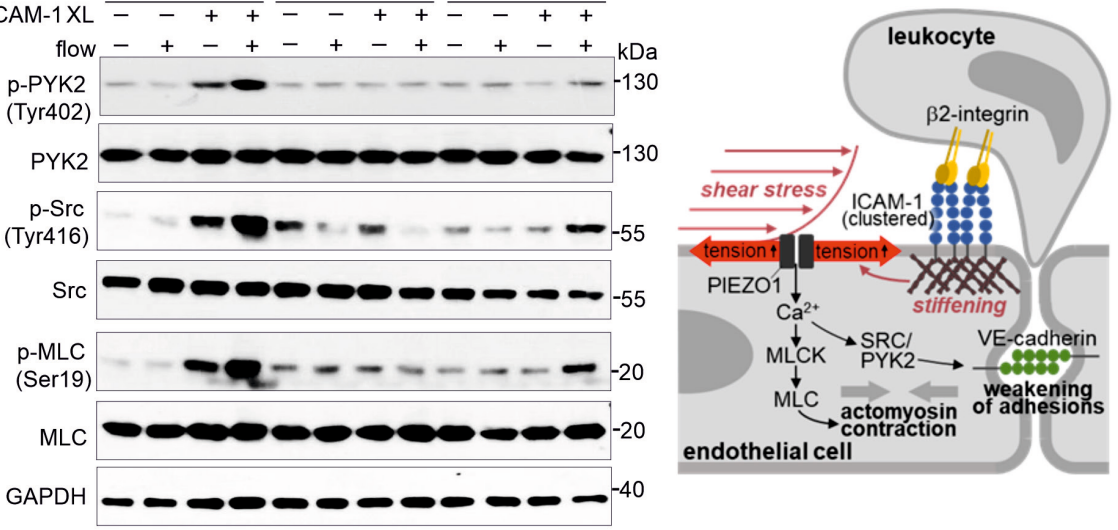

3 Figure 4. Flow and ICAM-1 clustering synergistically increase endothelial membrane 4 tension.

5 (a,b) Fluorescence lifetime $\tau_{1}$ images of FliptR in TNFa-activated HUVECs kept 6 under static conditions or in the presence of low flow (1.2 dynes $\left./ \mathrm{cm}^{2}\right)$ or exposed to 7 anti-ICAM-1 antibody beads or to anti-ICAM-1-crosslinking antibodies (ICAM-1 XL) 8 without or together with low flow. The color bar corresponds to lifetime in 9 nanoseconds (ns). Bar length: $15 \mu \mathrm{m}$. Corresponding lifetime mean values indicating 10 membrane tension are shown in the bar diagram (b; $n=40$ measurements from 5 
1 independent experiments). (c-f) HUVECs were preincubated without or with $10 \mu \mathrm{M}$ 2 cytochalasin $\mathrm{D}(\mathrm{CytoD})$ or $30 \mu \mathrm{M}$ blebbistatin (Bleb) (c,d) or were transfected with 3 control siRNA (siCtrl) or siRNA directed against the RNA encoding $\alpha$-actinin-4 4 (siACTN4) or cortactin (siCTTN) (e,f) and were exposed to low flow alone, anti-ICAM51 clustering antibodies (ICAM-1 XL) alone or both, and membrane tension was 6 determined using FliptR ( $\mathrm{c}, \mathrm{e} ; \mathrm{n}=20$ measurements from 3 independent experiments) 7 or immunoblot analysis of total and phosphorylated PYK2, SRC and MLC was 8 performed (d,f). Bar diagrams show lifetime mean values (c,e). (g) Schematic 9 representation showing how fluid shear stress exerted by the flowing blood and 10 leukocyte-induced ICAM-1 clustering synergistically activate PIEZO1 to induce 11 downstream signaling events resulting in opening of the endothelial barrier. Shown 12 are mean values \pm s.e.m.; ${ }^{*} \mathrm{P} \leq 0.05$; ${ }^{* *} \mathrm{P} \leq 0.01$; ${ }^{* * *} \mathrm{P} \leq 0.001$ (unpaired two-tailed $t$ 13 test). 
1 Extended Data

2
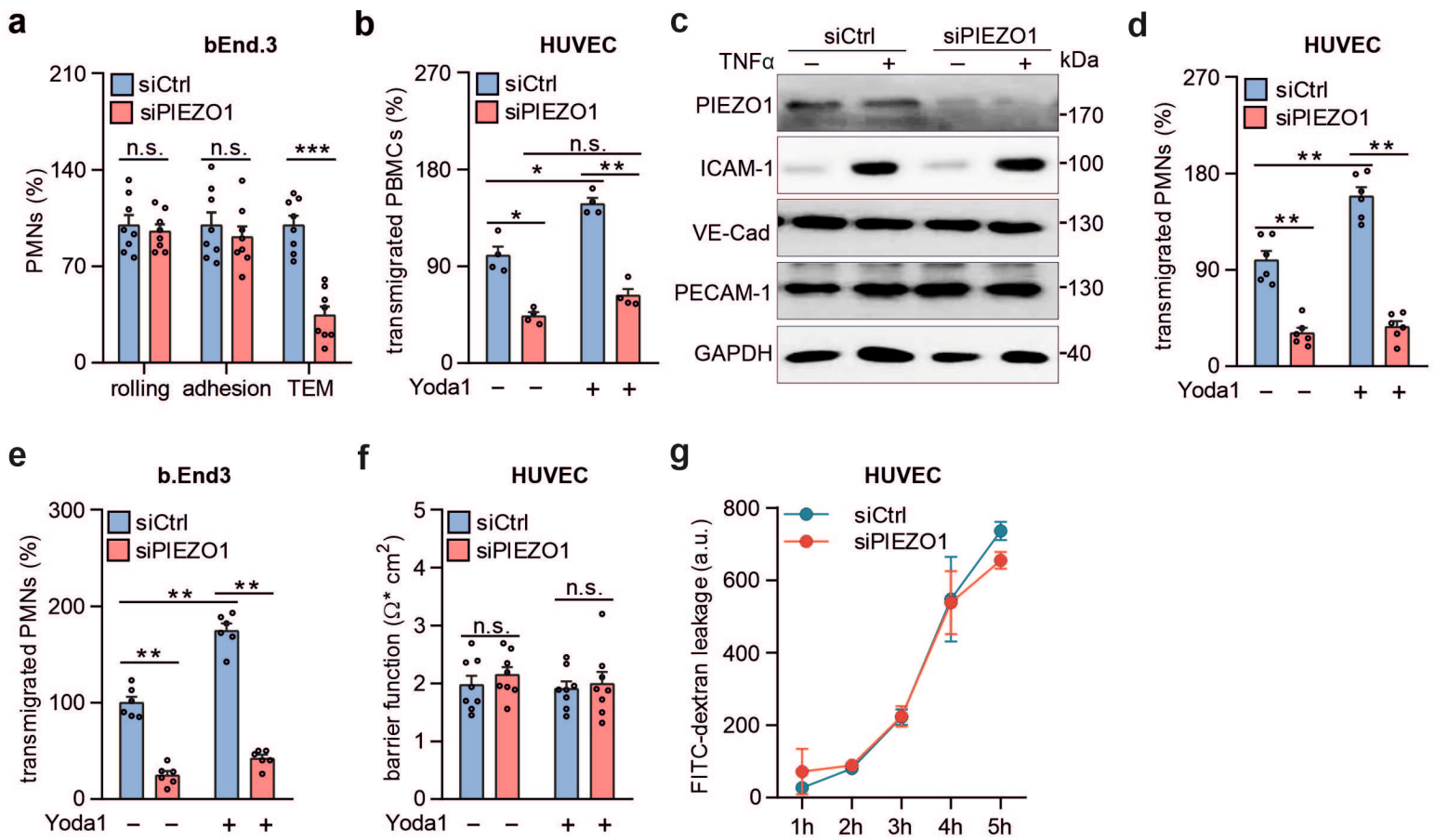

4 Ext. Data Figure 1. PIEZO1 mediates leukocyte transendothelial migration in vitro.

5 (a-g) The indicated endothelial cells were transfected with control (siCtrl) or PIEZO16 specific siRNA (siPIEZO1). (a) Rolling, adhesion and transmigration of mouse PMNs 7 ( $n=8$ per group) applied together with flow (1.2 dynes $/ \mathrm{cm}^{2}$ ) to a bEnd.3 cell 8 monolayer. Cells treated with control siRNA were set as $100 \%$. (b,d,e) 9 Transmigration of human PBMCs (b) ( $n=4$ per group), human PMNs (d) ( $n=6$ per group) or mouse PMNs (e) ( $n=6$ per group) across HUVECs (b,d) or bEnd.3 cells (e)

11 pre-treated without or with $1 \mu \mathrm{M}$ Yoda1 for $15 \mathrm{~min}$. (c) HUVECs were transfected with 12 control siRNA or siRNA directed against PIEZO1 and were incubated with $10 \mathrm{ng} / \mathrm{ml}$ 13 TNFa for $15 \mathrm{~h}$. Cells were then lysed and the indicated proteins were analyzed by 14 immunoblotting using the indicated antibodies. (f) HUVEC barrier integrity was assessed using an electric cell-substrate impedance sensing (ECIS) system in the absence or presence of $1 \mu \mathrm{M}$ Yoda1 ( $\mathrm{n}=8$ per group). (g) Paracellular permeability of the endothelial monolayer cultured in transwell plates was determined using $40 \mathrm{kDa}$ FITC-dextran ( $n=5$ per group; a.u., arbitrary units). Shown are mean values \pm s.e.m.; ${ }^{*} \mathrm{P} \leq 0.05 ;{ }^{* *} \mathrm{P} \leq 0.01 ;{ }^{* *} \mathrm{P} \leq 0.001$ (unpaired two-tailed $t$-test). 
a

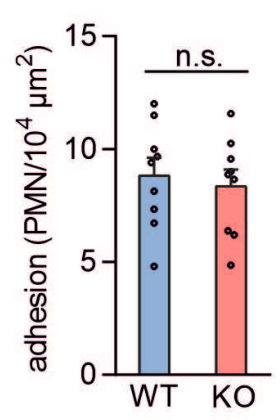

f

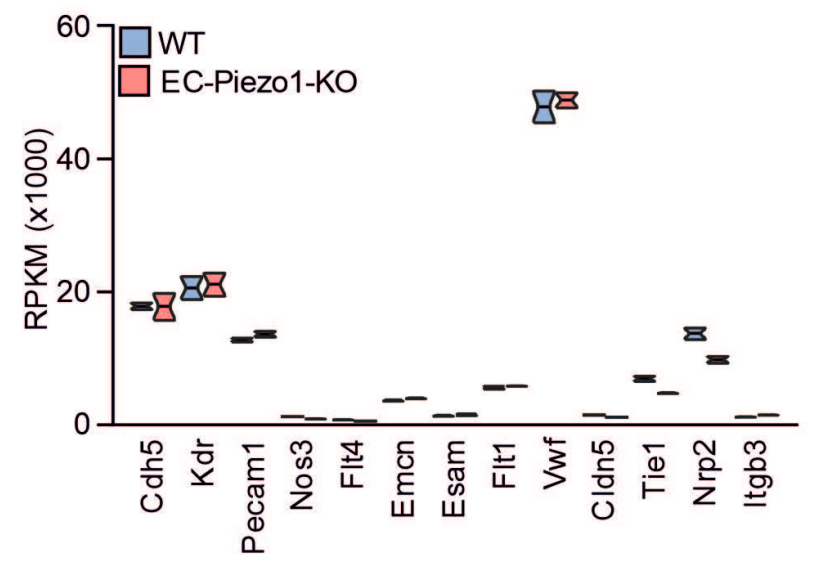

b

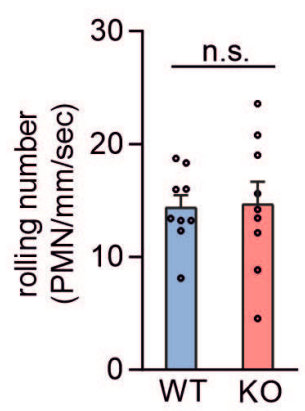

C

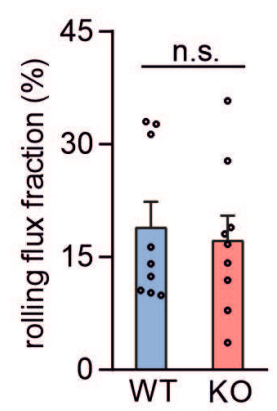

d

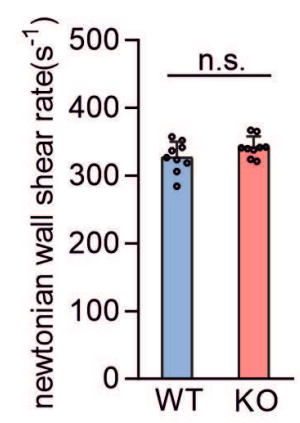

e

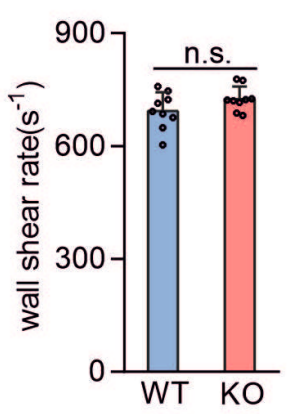

g

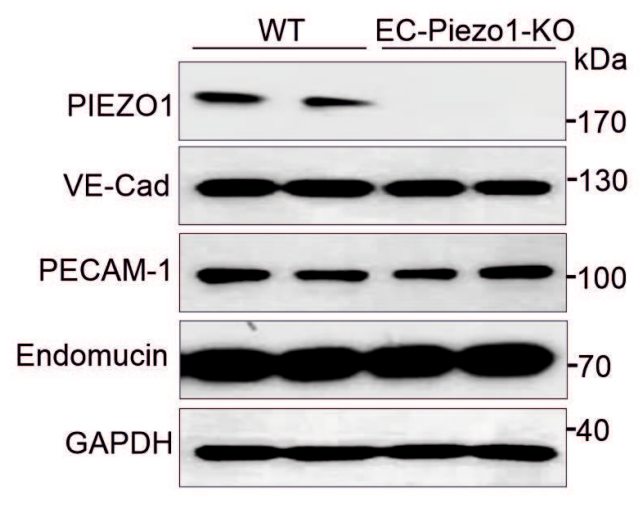

2 Ext. Data Fig. 2. Effect of endothelial PIEZO1 knock-out on hemodynamic 3 parameters, leukocyte rolling and adhesion as well as on expression of various 4 genes.

5 (a-e) WT and EC-Piezo1-KO mice were analyzed by intravital microscopy of

6 Cremaster venules 4 hours after injection of $50 \mathrm{ng} \mathrm{IL1} \beta$ for the number of adhering 7 PMNs (a), rolling PMNs per second per $\mathrm{mm}$ of vessel length (b), rolling flux fraction 8 (c), Newtonian wall shear rates (d) and wall shear rates (e) ( $n=9$ animals per group). 9 (f,g) Lung endothelial cells were isolated from wild-type (WT) and EC-Piezo1-KO 10 mice and analyzed by RNA-sequencing (f) or by immunoblotting (g). Shown are 11 mean values \pm s.e.m.; n.s., not significant (unpaired two-tailed $t$-test). 
a

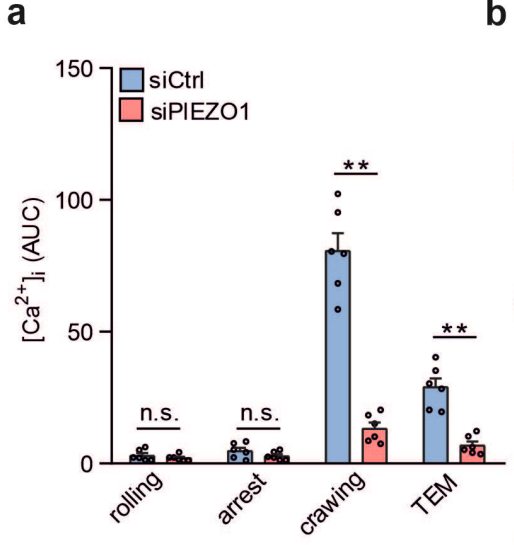

b

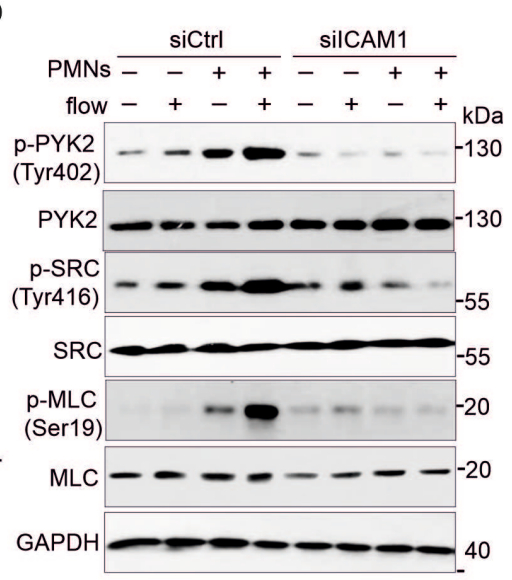

d

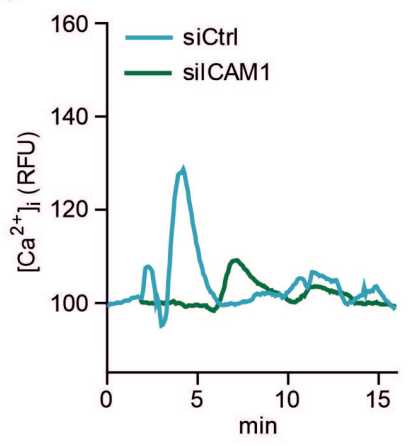

e

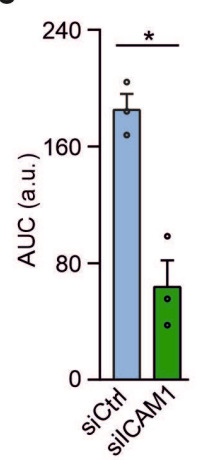

f

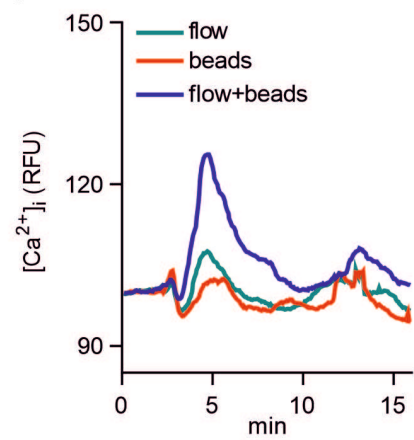

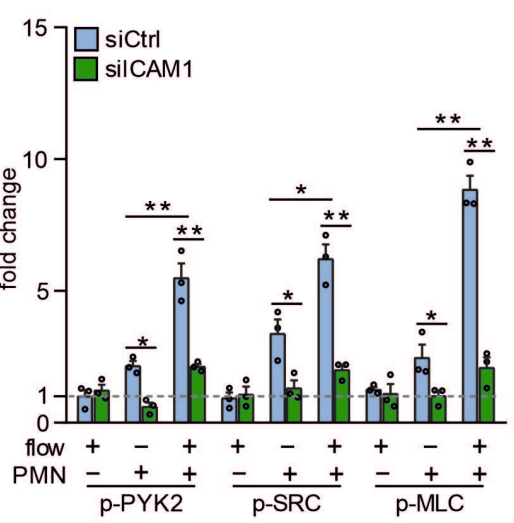

g

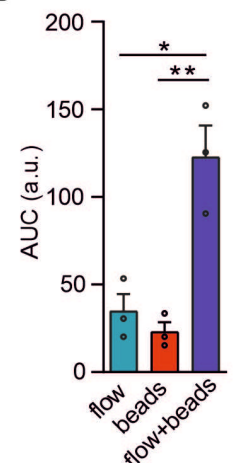

Ext. Data Fig. 3. Increases in $\left[\mathrm{Ca}^{2+}\right]_{\mathrm{i}}$ and downstream signaling mediated by PIEZO1 3 and ICAM-1.

4 (a) HUVECs loaded with Fluo4 were exposed to human neutrophils together with flow (1.2 dynes $\left./ \mathrm{cm}^{2}\right)$, and intracellular free $\left[\mathrm{Ca}^{2+}\right]_{i}$ was measured during different phases of PMN-endothelial cell interaction ( $n=6$ per group) (RFU, relative fluorescence units). (b-e) TNFa-activated HUVECs transfected with control siRNA (siCtrl) or siRNA directed against ICAM-1 were exposed to low flow alone, human PMNs alone or both, and immunoblot analysis of total and phosphorylated PYK2, SRC and MLC was performed. Immunoblot analysis of GAPDH served as control. The bar diagram (c)

11 shows the densitometric analysis of 3 independent experiments. Alternatively, the free $\left[\mathrm{Ca}^{2+}\right]_{i}$ was determined after loading of cells with Fluo4 $(\mathrm{d}, \mathrm{e})$. The bar diagram (e) 13 shows the area under the curve (AUC) of the $\left[\mathrm{Ca}^{2+}\right]_{i}$-trace from 3 independent 14 experiments (a.u., arbitrary units). (f,g) HUVECs were loaded with Fluo4 and 15 exposed to low flow (1.2 dynes $/ \mathrm{cm}^{2}$ ) alone, anti-ICAM-1 antibody-linked beads 16 (beads) or both flow and beads, and free $\left[\mathrm{Ca}^{2+}\right]_{i}$ was determined (RFU, relative 17 fluorescence units). The bar diagram shows the statistical evaluation of the area under the curve (AUC) ( $n=3)$ (a.u., arbitrary units). Shown are mean values \pm s.e.m.; ${ }^{*} \mathrm{P} \leq 0.05 ;{ }^{* *} \mathrm{P} \leq 0.01$ (unpaired two-tailed $t$-test). 
a

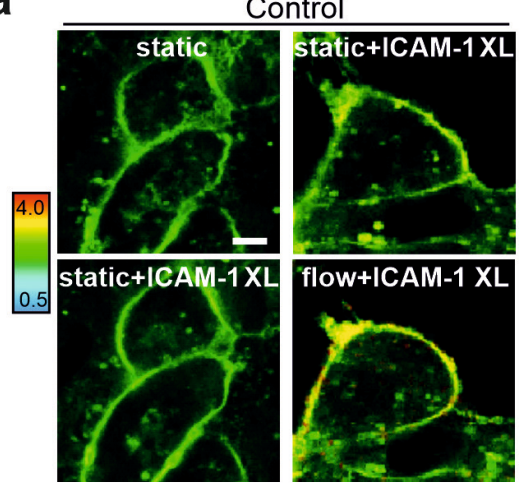

b

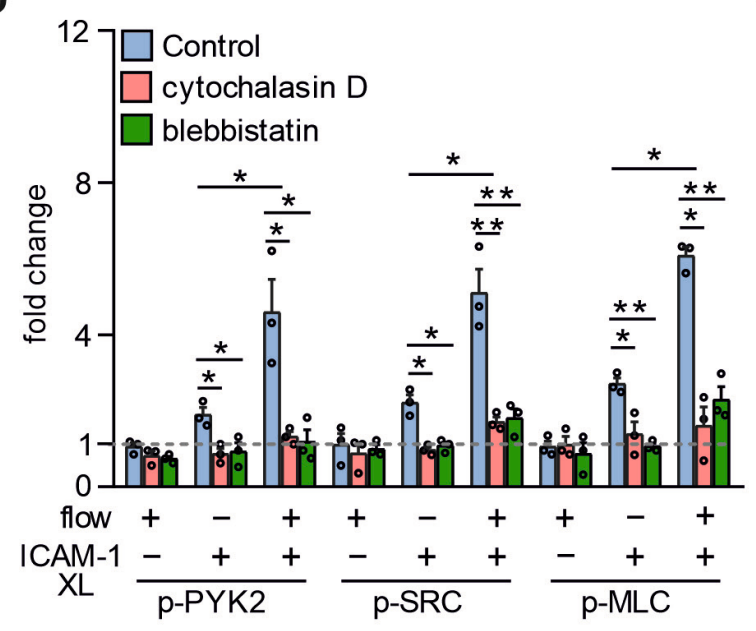

Cytochalasin D

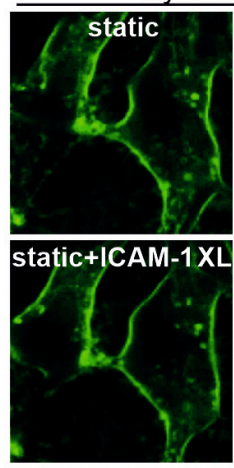

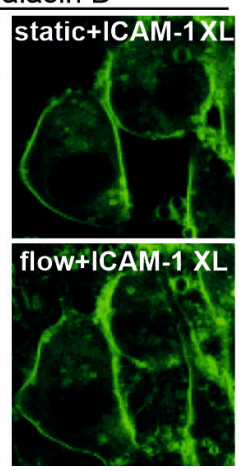

C

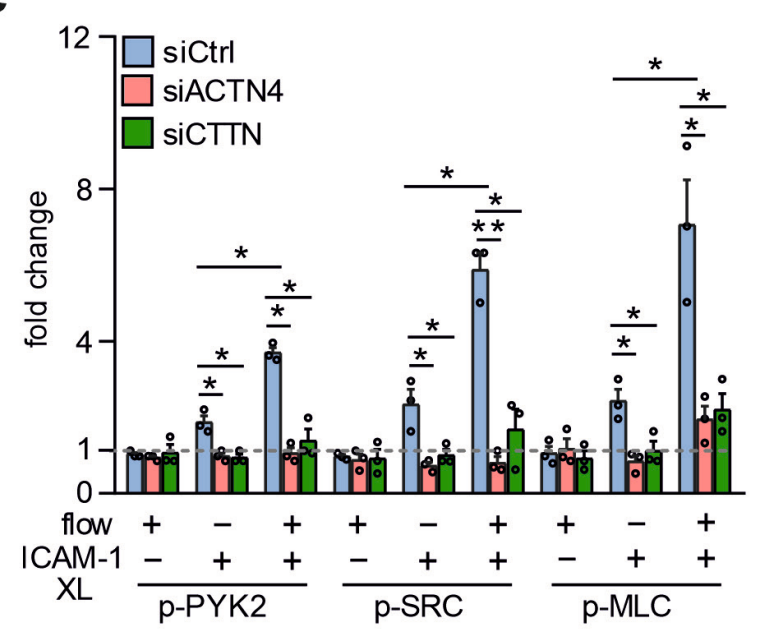

d

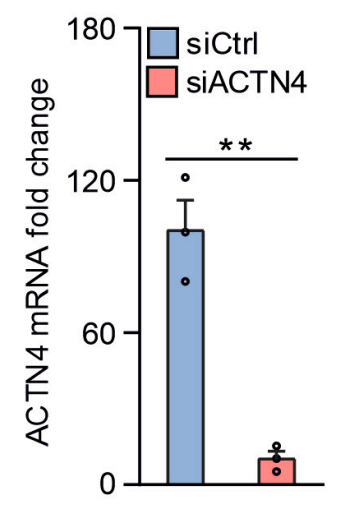

e

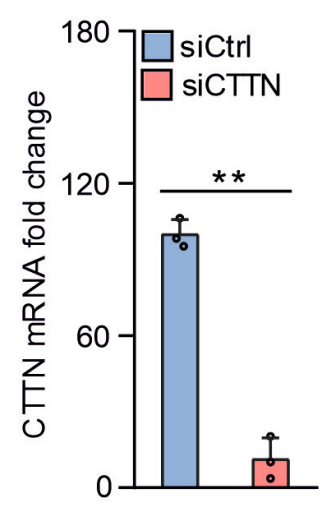

3 Ext. Data Fig. 4. Flow and ICAM-1 clustering synergistically induce downstream 4 signalling.

5 (a-c) HUVECs were preincubated without or with $10 \mu \mathrm{M}$ cytochalasin D (CytoD) or 30 $6 \mu \mathrm{M}$ blebbistatin (Bleb) $(\mathrm{a}, \mathrm{b})$ or were transfected with control siRNA (siCtrl) or siRNA 7 directed against the RNA encoding $\alpha$-actinin-4 (siACTN4) or cortactin (siCTTN) (c) 8 and were exposed to low flow alone, anti-ICAM-1 clustering antibodies (ICAM-1 XL) 9 alone or both and membrane tension was determined using FliptR (a), or immunoblot 
1 diagrams show the statistical analysis of 3 independently performed immunoblot 2 experiments. (d,e) Analysis of knock-down efficiency in HUVECs. HUVECs were 3 transfected with control siRNA (siCtrl) or siRNA directed against ACTN4 (d) or CTTN

4 (e). Knock-down efficiency was analyzed by qRT-PCR $(n=3)$. Shown are mean 5 values \pm s.e.m.; ${ }^{*} \mathrm{P} \leq 0.05 ;{ }^{* *} \mathrm{P} \leq 0.01$ (unpaired two-tailed $t$-test). 


\section{Methods}

\section{$2 \quad$ Cell culture and cell isolation}

3 Human umbilical vein endothelial cells (HUVECs) were obtained from Lonza and

4 cultured with EGM-2 (Lonza) supplemented with $5 \%$ fetal bovine serum (FBS)

5 (Lonza). Only confluent cells at passage $\leq 4$ were used in experiments. THP-1

6 monocyte cells were obtained from Sigma (Cat. No. 88081201) and were cultured in

7 RPMI 1641 medium (Invitrogen) supplemented with $2 \mathrm{mM}$ glutamine and 10\% FBS.

8 The bEnd.3 cell line was obtained from ATCC and cells were cultured in DMEM

9 medium (Invitrogen) supplemented with 10\% FBS. Primary mouse lung endothelial

10 cells (MLECs) were isolated using CD31-labelled dynabead (Miltenyi Biotec, Cat. No.

11 130-097-418) and were further purified by FACS using anti-CD144-PE antibodies

12 (BD Biosciences, Cat. No. 562243) as described previously ${ }^{51,52}$. Human

13 polymorphonuclear leukocytes (PMNs) and peripheral blood mononuclear cells

14 (PBMC) were isolated from peripheral blood by density gradient centrifugation using

15 Histopaque 1077 and 1119 (Sigma-Aldrich) and were resuspended in Hanks

16 balanced salt solution (HBSS) with 20 mM HEPES ( $\mathrm{pH} \mathrm{7.4)} \mathrm{and} 0.5 \%$ human serum

17 albumin (Sigma-Aldrich) ${ }^{53,54}$. Mouse PMNs from murine femora and tibiae were 18 isolated with an EasySep Mouse Neutrophil Enrichment Kit (STEMCELL

19 Technologies, Canada) according to manufacturer's instructions. The PMNs were 20 resuspended in Hanks balanced salt solution (HBSS) containing 20 mM HEPES (pH $21 \quad 7.4$ ) and $0.5 \%$ fetal calf serum and were immediately used for transmigration assays.

\section{3 siRNA-mediated knockdown}

24 Cells were transfected with siRNAs using Opti-MEM and Lipofectamine RNAiMAX 25 (Invitrogen) as described previously ${ }^{55}$. For transfection of cells in ibidi flow chambers, 
1 Fisher) and incubated for 30 minutes at room temperature. The mixture was then

2 added to $100 \mu \mathrm{l}$ of cell culture medium, and cells in the flow chamber (ibidi $\mu$-slide I)

3 were covered by the medium. The medium was changed after 6 hours. 18 hours later,

4 the siRNA transfection was repeated, and experiments were performed 48 hours

5 later. For transfection of cells in 96-transwell plates (Corning), 2.5 pmoles siRNA in

$610 \mu \mathrm{l}$ were prepared and added to $50 \mu \mathrm{l}$ cell culture medium. siRNAs against PIEZO1

7 and ICAM1 were from Sigma-Aldrich, and siRNAs against ACTN4 and CTTN were

8 from Genepharma. The targeted sequences of siRNAs directed against RNAs

9 encoding PIEZO1, ICAM-1, a-actinin-4 and cortactin were: PIEZO1 (human), 5'-

CCAAGTACTGGATCTATGT-3', 5'-GCAAGTTCGTGCGCGGATT-3', and 5'-

11 AGAAGAAGATCGTCAAGTA-3'; ICAM-1 (human), 5'-CAGCGGAAGATCAAGAAAT-

12 3', 5'-CCGAGCTCAAGTGTCTAAA-3', 5'-CAACCAATGTGCTATTCAA-3'; a-actinin-4

13 (human) 5'-CCACATCAGCTGGAAGGATGGTC-3', 5'-

14 GCAGCAGCGCAAGACCTTC-3'; cortactin (human) 5'-CCAGGAGCATATCAACATA15 3'; 5'-GCAACTTATTGTATCTGAA-3.

SiRNAs used for the screen were pools of siRNAs of a customized siRNA library (Sigma) directed against 360 genes encoding transmembrane proteins enriched in HUVECs. Only siRNAs resulting in suppression of expression levels to less than $25 \%$ of control levels as determined by quantitative RT-PCR were used.

\section{In vitro transmigration assay}

HUVECs or MLECs were seeded at $1.5 \times 10^{4}$ cells in $100 \mu \mathrm{l}$ and were cultured on collagen-coated 96-transwell plates with polyester membranes of $8 \mu \mathrm{m}$ pore size (Corning) until reaching confluency and were then incubated with $10 \mathrm{ng} / \mathrm{ml}$ recombinant TNFa (PeproTech, Cat. No. 300-01A) for 16 hours prior to the assay. For transmigration experiments, the medium of the upper compartment was removed 
1 and $8 \times 10^{3}$ calcein-AM-labelled PMNs were added in $50 \mu \mathrm{l}$ of HBSS alone or in the

2 presence of the indicated substances. 30 min later, transmigrated PMNs on the lower

3 side of the filter were imaged (Olympus IX81 or Zeiss Axio Observer Z1) and

4 quantified with ImageJ. The screen to identify potential transmembrane proteins that

5 mediate trans-endothelial migration of THP-1 cells was performed in a 96-well format.

6 The ratio of transmigration for each condition was defined as transmigration of THP-1

7 cells after transfection of HUVECs with an individual siRNA pool divided by the 8 transmigration after transfection with control siRNA. For transmigration assay under

9 flow, $1.5 \times 10^{4}$ HUVECs were cultured per channel in a fibronectin-coated ibidi $\mu$ -

10 Slide I using a parallel-plate flow chamber and stimulated with $10 \mathrm{ng} / \mathrm{ml}$ recombinant

11 TNFa (PeproTech) 16 hours prior to the assay. The flow chamber was perfused with

12 HBSS at a constant shear flow $\left(1.2\right.$ dyne $\left./ \mathrm{cm}^{2}\right)$ using a computer controlled air 13 pressure pump (ibidi) for $15 \mathrm{~min}$. PMNs were subsequently injected into the perfusion medium and the transmigration process was recorded for $30 \mathrm{~min}$ at 0.5 frames/s using a IX81 (Olympus) microscope at $37^{\circ} \mathrm{C}$ in the presence of $5 \% \mathrm{CO}_{2}$. Percentage of rolling, adhering, crawling and transmigrated PMNs were manually quantified using Image J as described previously ${ }^{56}$. Rolling cells were defined as those that move more than 1 cell diameter within $10 \mathrm{~s}$, while adhering cells were those that moved less than 1 cell diameter within $5 \mathrm{~s}$. Cells transmigrating the endothelial monolayer were directly visualized and crawling was defined as the period between adhesion 21 and transmigration.

\section{Immunoblot analysis}

24 Cells were lysed in cell lysis buffer (Cell Signaling, Cat. No. 9803) containing $1 \%$ triton X-100 or in RIPA buffer (Cell Signaling, Cat. No. 9806) supplemented with protease and phosphatase inhibitors (Cell Signaling, Cat. No. 5872). Lysates were 
1 centrifuged at $10,000 \times \mathrm{g}$ at $4^{\circ} \mathrm{C}$ for 10 minutes. Supernatants were then subjected to

2 SDS-PAGE and transferred to nitrocellulose membranes. Membranes were probed

3 with primary and HRP-conjugated secondary antibodies (Cell Signaling Technology,

4 Cat. No. 8884 and 7076, respectively) and were developed using the ECL detection 5 system (Pierce).

8 For the determination of the intracellular $\mathrm{Ca}^{2+}$ concentration, endothelial cells were 9 loaded with $5 \mu \mathrm{M} \mathrm{Ca}^{2+}$-sensitive dye Fluo-4 AM (Molecular Probes, Cat. No. F14201) in HBSS supplemented with $20 \mathrm{mM}$ HEPES for $30 \mathrm{~min}$ at $37^{\circ} \mathrm{C}$ and were then

11 washed with HBSS 3 times at room temperature. Live-cell images were acquired with 12 an IX81 microscope (Olympus) at a frequency of $1 \mathrm{~Hz}$. Fluorescence intensity was measured using a FlexStation 3 (Molecular Devices).

\section{FITC-dextran permeability assay} pore size, Corning) and were cultured with daily medium changes until reaching confluency. For knockdown experiments, 8000 cells were transfected using confluency. For knockdown experiments, 8000 cells were transfected using

\section{Transendothelial electrical resistance measurement}

Endothelial barrier function was assessed in real-time by continuously recording change in trans-endothelial electrical resistance using the ECIS ZTheta system (Applied BioPhysics) as described previously ${ }^{57}$. In brief, 40,000 HUVECs were seeded per well of a 96W1E + PET electrode plate coated with $1 \%$ gelatin (Applied BioPhysics). HUVEC barrier integrity was analyzed after $48 \mathrm{~h}$ when cells had formed a confluent monolayer.

$1.5 \times 10^{4}$ HUVECs were seeded per well of a collagen-coated transwell plate $(3 \mu \mathrm{m}$ 
1 Lipofectamine RNAiMAX with the indicated siRNAs. For permeability assay, the

2 medium of the upper insert was removed and replaced with medium containing 250

$3 \mu \mathrm{g} / \mathrm{ml}$ fluorescein isothiocyanate (FITC)-conjugated dextran (relative molecular mass

$440 \mathrm{kDa}$; Molecular Probes). The permeability was determined by passage of FITC-

5 dextran through the endothelial monolayer into the lower chamber using FlexStation-

63 (Molecular Devices).

7

\section{Determination of cell membrane tension}

Membrane tension was determined as previously described ${ }^{58,59}$. Briefly, endothelial cells cultured in ibidi flow chambers or collagen-coated 8-well glass chambers (Nunc) were stimulated with TNFa overnight and incubated with $1 \mu \mathrm{M}$ of the membrane tension probe Flipper-TR® (Tebu-bio, Cat. No. SC020) for 30 min at $37^{\circ} \mathrm{C}$. Cells were then washed 3 times with HBSS and subjected to flow $\left(1.2 \mathrm{dynes} / \mathrm{cm}^{2}\right)$ alone, PMNs alone, or flow together with PMNs, anti-ICAM-1 beads or ICAM-1-clustering antibodies (see below) and imaged with a Leica-SP8 FLIM microscope. Excitation was performed using a pulsed $488 \mathrm{~nm}$ laser (Laser kit WLL2+pulse picker, Leica Microsystems) operating at $80 \mathrm{MHz}$, and the emission signal was collected from 549 to $651 \mathrm{~nm}$ with acousto-optical beam splitter (AOBS) using a gated hybrid (HyD SMD) detectors and a TimeHarp 300 TCSPC Module and Picosecond Event Timer (PicoQuant). SymPhoTime 64 software (PicoQuant) was then used to fit fluorescence decay data. To extract lifetime information, the photon histograms from membrane regions were fitted with a double exponential, and 2 fluorescence emission decay times ( $\mathrm{\tau} 1$ and $\mathrm{\tau} 2$ ) are extracted. The longest lifetime with the higher fit amplitude $\mathrm{\tau} 1$ is used to report membrane tension ${ }^{59}$. 


\section{ICAM-1 clustering}

2 For antibody-mediated clustering of ICAM-1, sheep-anti mouse IgG-coupled 3 dynabeads (Invitrogen, Cat. No. M280) were coated with mouse anti-human ICAM-1

4 antibody (R\&D Systems, Cat. No. BBIG-I1) or IgG1 control (R\&D Systems, Cat. No. experiments, antibody-coated beads were injected into the ibidi perfusion system containing HUVECs to induce ICAM-1 clustering on HUVECs under physiological flow conditions. Alternatively, ICAM-1 was ligated with $15 \mu \mathrm{g} / \mathrm{ml}$ mouse monoclonal

11 antibodies (R\&D Systems, Cat. No. BBIG-11) for 30 min, followed by washing and ICAM-1 cross-linking with $50 \mu \mathrm{g} / \mathrm{ml}$ mouse secondary antibody (R\&D Systems, Cat. No. AF007) for $20 \mathrm{~min}$ at $37^{\circ} \mathrm{C}$. Live cell imaging of membrane tension and buffer as described above.

\section{VE-cadherin internalization assay}

The endocytosis of VE-cadherin was assayed as described ${ }^{60}$. HUVECs were grown to confluency on collagen-coated eight-well glass chamber (Nunc) or fibronectincoated flow chambers (ibidi, $\mu$-slide I). Cells were stimulated with $10 \mathrm{ng} / \mathrm{ml}$ TNF $\alpha$ before they were incubated with $150 \mu \mathrm{M}$ chloroquine (Sigma, Cat. No. C6628). The antibody against VE-cadherin (Becton Dickinson, Cat. No. 555661) was dialyzed into the cell culture medium and incubated with cells for $1 \mathrm{~h}$ at $4{ }^{\circ} \mathrm{C}$. Free antibody was removed by rinsing cells in cold EGM-2 medium. Cells were switched back to $37^{\circ} \mathrm{C}$ 
1 and were incubated for 15 min with $1 \times 10^{4}$ freshly isolated PMNs per chamber

2 without or with flow $\left(1.2 \mathrm{dynes} / \mathrm{cm}^{2}\right)$. PMNs were then removed by rinsing of cells

3 three times with PBS. To remove cell surface-bound antibody while retaining the

4 internalized antibody, cells were washed with PBS (pH 2.7) containing $25 \mathrm{mM}$ glycine 5 and 5\% BSA for 15 min. Cells were then fixed with 4\% paraformaldehyde and 6 processed for permeabilization and immunofluorescence staining with secondary 7 antibodies Alexa Fluor 488-goat anti-mouse (Invitrogen, Cat. No. A28175), and DNA was stained with DAPI (Invitrogen, Cat. No. D1306). Fluorescence signals were detected with a confocal laser-scanning microscope (Leica SP8 or Olympus C2).

11 Mice

12 All mice were backcrossed onto a C57BL/6N background at least 10 times, and experiments were performed with littermates as controls. Male and female animals at an age of 8-12 weeks were used unless stated otherwise. Mice were housed under a 12-hour light-dark cycle with free access to food and water and under specific pathogen-free conditions. The generation of inducible endothelium-specific PIEZO1deficient mice (Tek-CreERT2;Piezo1 ${ }^{\text {fl/fl }}$ [EC-Piezo1-KO]) was described previously ${ }^{55}$.

18 To induce recombination, animals were injected on 5 consecutive days with $1 \mathrm{mg} / \mathrm{d}$ tamoxifen (Sigma) dissolved in corn oil, and 10-14 days later experiments were started. All animals which served as controls for tamoxifen-induced endothelial 21 specific animals were treated with the same amount of tamoxifen under the same conditions. All procedures of animal care and use in this study were approved by the

23 local animal welfare authorities and committees (Regierungspräsidium Darmstadt, 24 Germany and Ethical Committee of Xi'an Jiaotong University, China). 


\section{Intravital microscopy}

2 Mice were anesthetized with an intraperitoneal injection of $125 \mathrm{mg} / \mathrm{kg}$ ketamine

3 hydrochloride (Zoetis) and $12.5 \mathrm{mg} / \mathrm{kg}$ xylazine (Bayer). Cremaster muscle was 4 prepared, and intravital microscopy was carried out as previously described ${ }^{61}$. 5 Postcapillary venules with a diameter of 20 to $40 \mu \mathrm{m}$ were chosen for recording using 6 an intravital upright microscope (Zeiss Axio Examiner Z.1) with a 20x W.Plan 7 Apochromat 1.0 numerical aperture saline immersion objective (Zeiss). Inflammation

\section{TNFa-induced peritonitis and flow cytometry}

Wild-type or EC-Piezo1-KO mice were injected intraperitoneally with $100 \mu$ PBS containing no or $500 \mathrm{ng}$ TNFa prewarmed to $37^{\circ} \mathrm{C}$. After $60 \mathrm{~min}$, animals were killed and cells in the peritoneal cavity were collected by flushing with $5 \mathrm{ml}$ ice-cold PBS. Peritoneal cells were filtered using a $70-\mu \mathrm{m}$ strainer and analyzed by flow cytometry (BD FACS Canto II). The following antibodies were used: FITC conjugated antimouse CD11b (BioLegend, Cat. No. 101205) and APC-conjugated anti-mouse Ly6G (BioLegend, Cat. No. 127614).

\section{In vivo vascular permeability assay}

A modified Miles assay to determine vascular permeability in the skin was performed as described ${ }^{62}$. Mice were anaesthetized by isoflurane and shaved 2 days before the assay. Animals were then intraveneously injected with $150 \mu \mathrm{l}$ of $1 \%$ Evans blue solution, and $15 \mathrm{~min}$ later $20 \mu \mathrm{l}$ of VEGF (100 ng/ml, PreproTech) or histamine (100 $\mu \mathrm{M}$, Sigma-Aldrich) in PBS was injected intradermally at the shaved back skin of the mice. $20 \mu \mathrm{l}$ of PBS was injected as control. 30 min later mice were killed by $\mathrm{CO}_{2}$, 
1 perfused with PBS, and the area around the injection site was dissected. The

2 extravascular dye was then extracted by incubation with formamide at $56^{\circ} \mathrm{C}$ for 2

3 days. The degree of vascular leakage was determined by measuring 4 spectrophotometrically at $620 \mathrm{~nm}$ the ratio of Evans blue light absorption in test 5 samples and control samples.

\section{Ear dermatitis induced by croton oil}

The method of croton oil-induced dermatitis was perfomed as previously described 17,63,64. Briefly, wild-type or EC-Piezo1-KO mice were treated on the inner surface of the right ear with croton oil (Sigma-Aldrich, $2 \% \mathrm{v} / \mathrm{v}$ in a $4: 1$ mixture of acetone and olive oil). The left ear was treated with vehicle solution as control. Mice were killed 6 $\mathrm{h}$ later, and both ears were harvested and fixed overnight in $4 \%$ paraformaldehyde at $4^{\circ} \mathrm{C}$ in PBS. Ears were then permeabilized with $0.5 \%$ Triton $\mathrm{X}-100,5 \%$ BSA in PBS and incubated in blocking solution (0.5\% Triton $\mathrm{X}-100,5 \% \mathrm{BSA}$ in PBS) for $24 \mathrm{~h}$ at $4^{\circ} \mathrm{C}$. Thereafter samples were incubated with anti-PECAM-1 (BD, clone MEC 13.3, Cat. No. 550274), anti-MRP-14 (R\&D Systems, Cat. No. AF2065), or anti-collagen IV (Bio-Rad, Cat. No. 2150-1470) overnight at $4^{\circ} \mathrm{C}$. Alexa Fluor 488 donkey anti-goat, Alexa Fluor 594 goat anti-rabbit and Alexa Fluor 647 chicken anti-rat (Thermo Fisher Scientific, Cat. No. A11055, A11037 and A21472, respectively) secondary antibodies were used. Tissue was mounted in fluorescent mounting medium (Polysciences, Cat. No. 18606-5), Z-stack projection were acquired using a Leica SP5 or SP8 confocal microscope and image analysis was performed with Imaris (Bitplane) and ImageJ.

\section{Other reagents and antibodies}

Yoda1 (Cat. No. 5586) was from Tocris Bioscience. Cytochalasin D (Cat. No. C8273), Blebbistatin (Cat. No. B0560) and PF431396 (Cat. No. PZ0185) were from 
1 Sigma. PP2 (Cat. No. 529576) was from Merck Chemicals. Anti-PIEZO1-antibody

2 was from Proteintech (Cat. No. 15939-1-AP). Anti-GAPDH (Cat. No. \#5174), anti-

3 PYK2 (Cat. No. \#3292), anti-p-PYK2(Tyr402, Cat. No. \#3291), anti-SRC (Cat. No.

4 \#2109), anti-p-SRC (Tyr416, Cat. No. \#6943), anti-MLC (Cat. No. \#3672) and anti-p-

$5 \quad$ MLC (Ser19, Cat. No. \#3675) were from Cell Signaling Technology. Anti-endomucin-

6 antibody was from Santa Cruz (Cat. No. sc-65495).

$7 \quad$ Statistical analysis

8 Trial experiments or experiments done previously were used to determine sample

9 size with adequate statistical power. Samples were excluded in cases where

RNA/cDNA quality or tissue quality after processing was poor (below commonly

11 accepted standards). Data are presented as means \pm SEM. Comparisons between 2

12 groups were performed with unpaired 2-tailed Student's $t$ test, and multiple group comparisons at different time points were performed by 2-way ANOVA followed by Bonferroni's post hoc test. $\mathrm{P} \leq 0.05$ was considered to be statistically significant.

\section{Data availability}

All data are available from the corresponding authors upon request.

\section{References (Methods)}

51 Wang, S. et al. $\mathrm{P} 2 \mathrm{Y}_{2}$ and $\mathrm{Gq} / \mathrm{G}_{11}$ control blood pressure by mediating endothelial mechanotransduction. J Clin Invest 125, 3077-3086 (2015).

52 Kaur, H. et al. Single-cell profiling reveals heterogeneity and functional patterning of GPCR expression in the vascular system. Nat Commun 8, 15700, doi:10.1038/ncomms15700 (2017).

53 Frye, M. et al. Interfering with VE-PTP stabilizes endothelial junctions in vivo via Tie-2 in the absence of VE-cadherin. $J$ Exp Med 212, 2267-2287, doi:10.1084/jem.20150718 (2015).

54 Braun, L. J. et al. Platelets docking to VWF prevent leaks during leukocyte extravasation by stimulating Tie-2. Blood 136, 627-639, doi:10.1182/blood.2019003442 (2020). 
55 Wang, S. et al. Endothelial cation channel PIEZO1 controls blood pressure by mediating flow-induced ATP release. J Clin Invest 126, 4527-4536, doi:10.1172/JCl87343 (2016).

56 Heemskerk, N. et al. F-actin-rich contractile endothelial pores prevent vascular leakage during leukocyte diapedesis through local RhoA signalling. Nat Commun 7, 10493, doi:10.1038/ncomms10493 (2016).

57 Stanicek, L. et al. Long non-coding RNA LASSIE regulates shear stress sensing and endothelial barrier function. Commun Biol 3, 265, doi:10.1038/s42003-0200987-0 (2020).

58 Wang, S. et al. Adipocyte Piezo1 mediates obesogenic adipogenesis through the FGF1/FGFR1 signaling pathway in mice. Nat Commun 11, 2303, doi:10.1038/s41467-020-16026-w (2020).

59 Colom, A. et al. A fluorescent membrane tension probe. Nat Chem 10, 11181125, doi:10.1038/s41557-018-0127-3 (2018).

60 Wessel, F. et al. Leukocyte extravasation and vascular permeability are each controlled in vivo by different tyrosine residues of VE-cadherin. Nat Immunol 15, 223-230, doi:10.1038/ni.2824 (2014).

61 Artz, A., Butz, S. \& Vestweber, D. GDF-15 inhibits integrin activation and mouse neutrophil recruitment through the ALK-5/TGF-betaRII heterodimer. Blood 128, 529-541, doi:10.1182/blood-2016-01-696617 (2016).

62 Mikelis, C. M. et al. RhoA and ROCK mediate histamine-induced vascular leakage and anaphylactic shock. Nat Commun 6, 6725, doi:10.1038/ncomms7725 (2015).

63 Tubaro, A., Dri, P., Delbello, G., Zilli, C. \& Della Loggia, R. The croton oil ear test revisited. Agents Actions 17, 347-349, doi:10.1007/BF01982641 (1986).

64 Schenkel, A. R., Chew, T. W. \& Muller, W. A. Platelet endothelial cell adhesion molecule deficiency or blockade significantly reduces leukocyte emigration in a majority of mouse strains. J Immunol 173, 6403-6408, doi:10.4049/jimmunol.173.10.6403 (2004). 


\section{Figures}

a

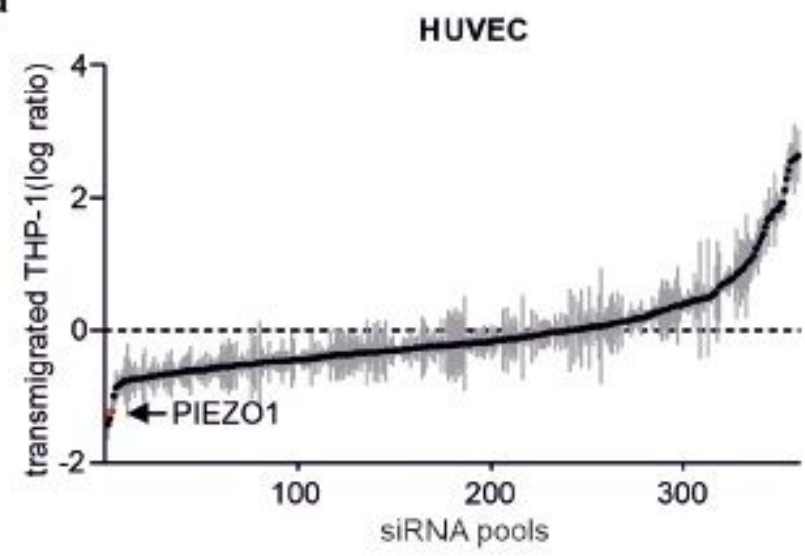

b

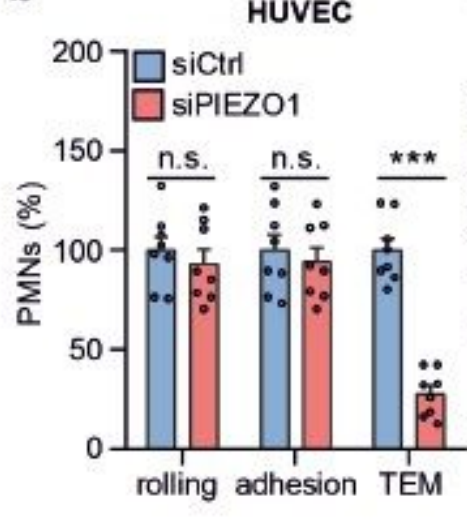

C

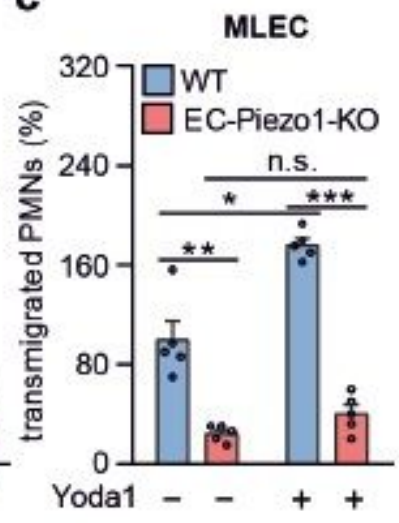

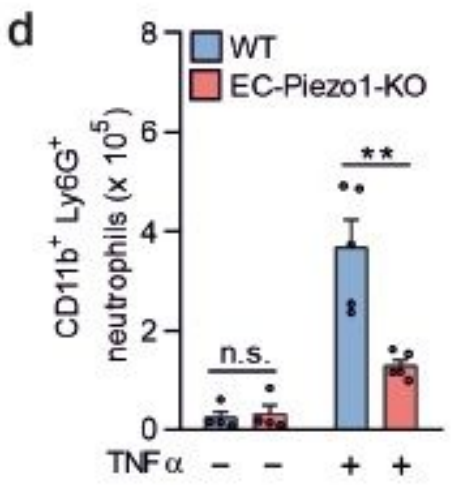

g

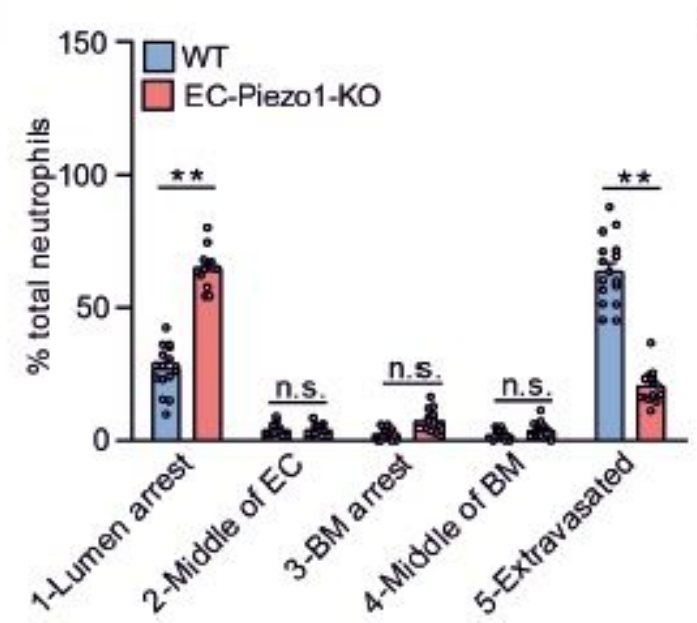

e

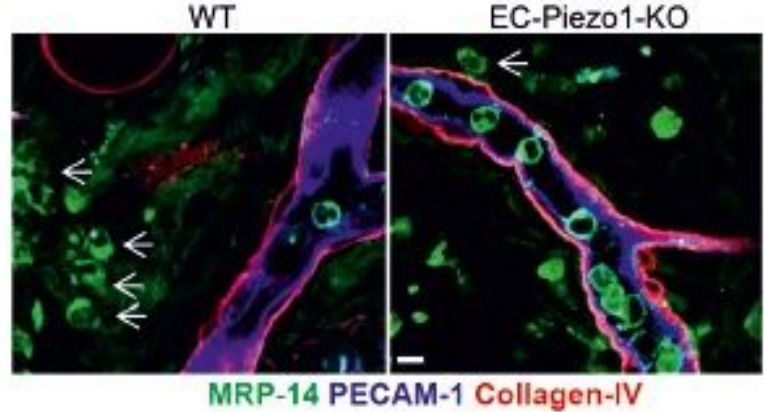

f

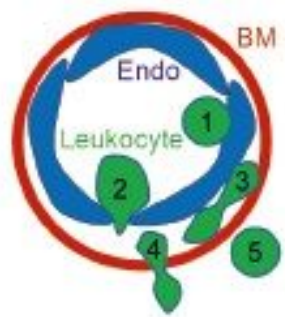

h

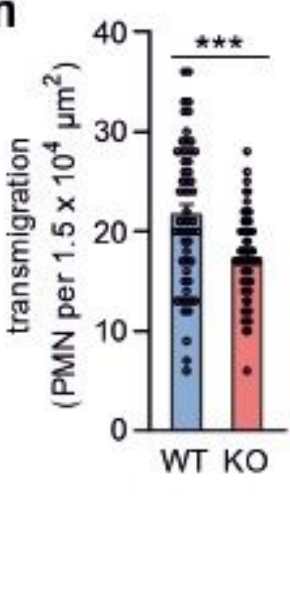

i

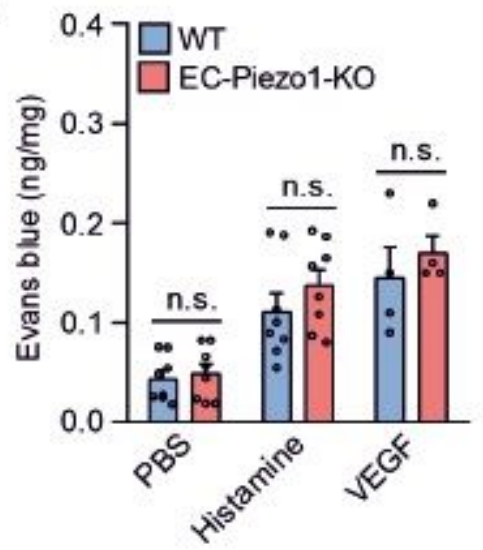

Figure 1

PIEZO1 mediates leukocyte transendothelial migration in vitro and in vivo. (a) HUVECs pretreated with 10 $\mathrm{ng} / \mathrm{ml}$ TNFa were transfected with 360 siRNAs pools against RNAs encoding transmembrane proteins expressed in endothelial cells and were then exposed to THP-1 monocytic cells for 3 hours. Shown is the ratio of THP-1 cells which transmigrated the HUVEC monolayer transfected with a particular siRNA pool and with control siRNA. The plot shows the ranked average ratios of three independent experiments. (b) 
HUVECs were transfected with control (siCtrl) or PIEZ01-specific siRNA (siPIEZO1), and rolling, adhesion and transmigration of human PMNs applied together with flow $(1.2$ dynes $/ \mathrm{cm} 2)$ were analyzed $(\mathrm{n}=8$ per group). Cells treated with control siRNA were set as $100 \%$. (c) Mouse lung 13 endothelial cells (MLECs) were isolated from wild-type (WT) and EC-Piezo1-KO mice and transmigration of mouse PMNs was determined after pretreament without or with $1 \mu \mathrm{M}$ Yoda 1 for $15 \mathrm{~min}(\mathrm{n}=5)$. (d) Wild-type (WT) and endothelium-specific PIEZO1 deficient mice (EC-Piezo1-KO) were injected intraperitoneally with PBS or $500 \mathrm{ng}$ ofTNFa, and the number of peritoneal CD11b+;Ly6G+ neutrophils was determined byflow cytometry ( $\mathrm{n}=4$ mice (-TNFa); $\mathrm{n}=5$ mice (+TNFa)). (e-g) Wild-type (WT) and EC- Piezo1-KO mice were treated with croton oil on one ear. $6 \mathrm{~h}$ later, animals were killed and ears were immunostained as whole mounts with antibodies against PECAM-1(blue, endothelium), collagen-IV (red, basement membrane) and MRP14 (green, neutrophil). Arrows indicate neutrophils. Scale bar: $10 \mu \mathrm{m}$. (e) Representative images of stained ears. (f) Schematic drawing illustrating the criteria to delineate the 5 positions in which leukocyte are found during extravasation. (g) Distribution pattern of neutrophil positions relative to the endothelium and basement membrane ( $n=16$ mice (WT); $n=14$ mice (EC-Piezo1-KO), 3-5 vessels were analyzed per animal). (h) WT and EC-Piezo1-KO mice were analyzed by intravital microscopy of cremaster venules 4 hours after injection of $50 \mathrm{ng}$ IL-1 $\beta$ for extravasated leukocytes ( $\mathrm{n}=9$ mice per group; 4-10 measurements per animal). (i) Evans blue extravasation was assessed after subcutaneous injection of $20 \mu \mathrm{l}$ of PBS without or with $100 \mu \mathrm{M}$ of histamine or $100 \mathrm{ng} / \mathrm{ml}$ of VEGF ( $\mathrm{n}=8$ mice (PBS and histamine); $\mathrm{n}=4$ mice

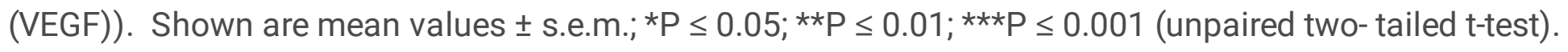



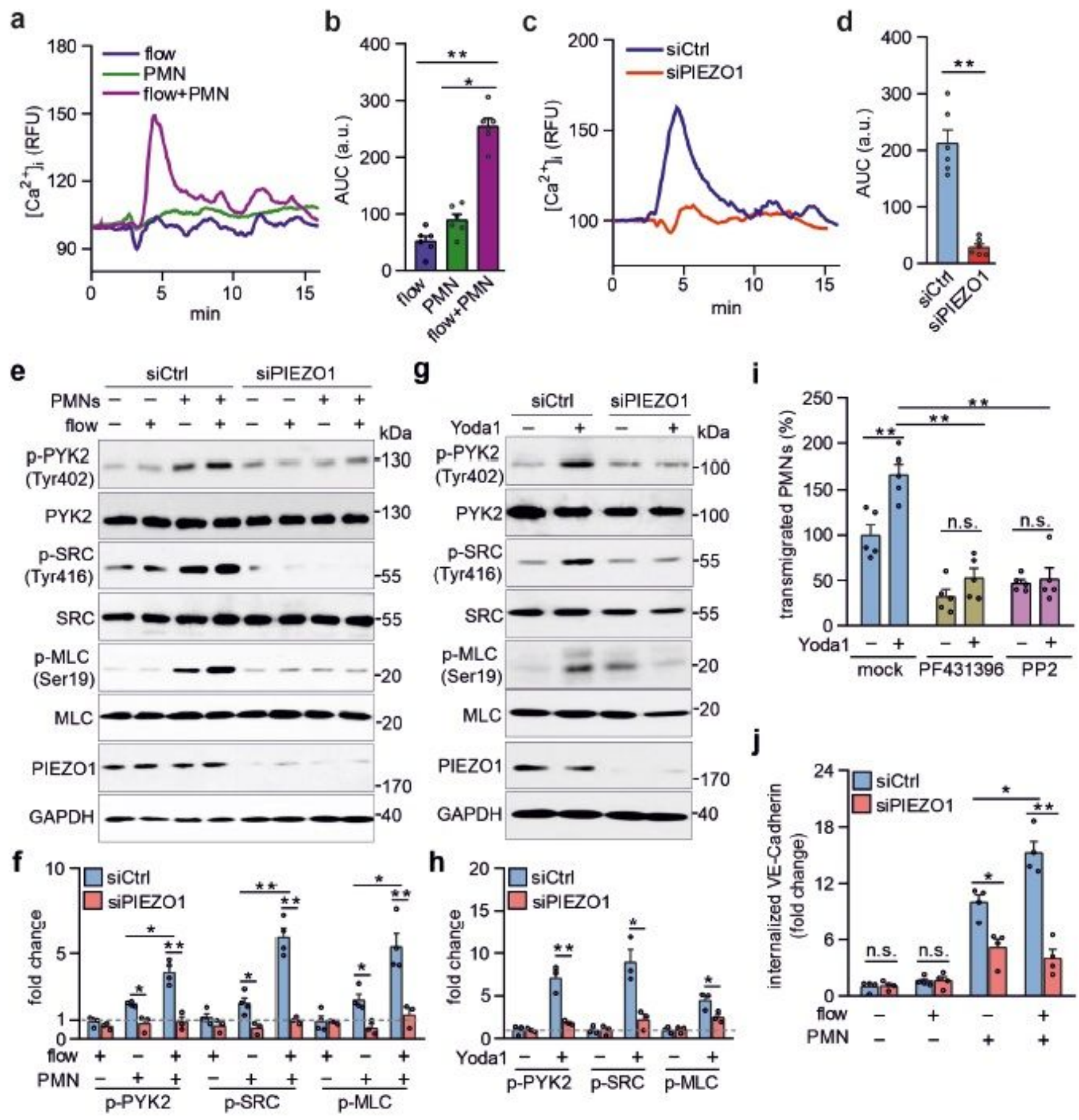

\section{Figure 2}

Leukocytes and flow synergistically induce PIEZO1 activation to stimulate endothelial downstream signaling.(a-d) Untransfected HUVECs (a,b) or HUVECs transfected with control (siCtrl) or PIEZO1-specific siRNA (siPIEZ01) (c,d) were preactivated with TNFa, loaded with Fluo-4 and were then exposed to PMNs alone, to low flow $(1.2$ dynes/cm2) alone or to both $(a, b)$ or to PMNs and low flow together $(c, d)$. [Ca2+]i was determined as fluorescence intensity (RFU, relative fluorescence units) ( $a, c)$. b and d show the area under curve (AUC) of the [Ca2+]i-trace from 6 independent experiments (a.u., arbitrary units). (e-h) Immunoblot analysis of total and phosphorylated PYK2, SRC and MLC in lysates of TNFa-activated 
HUVECs transfected with control siRNA (siCtrl) or siRNA directed against PIEZO1 and incubated without or with human PMNs in the absence or presence of low flow (1.2 dynes/cm2) (e) or without or with $5 \mu \mathrm{M}$ Yoda1 (g). Immunoblot analysis of PIEZO1 and GAPDH served as controls. Bar diagrams (f,h) show the densitometric analysis of independent experiments. (i) Transmigration of human PMNs across TNFaactivated HUVECs preincubated for 30 min with the PYK2 and SRC inhibitors PF431396 (10 $\mu \mathrm{M})$ and PP2 $(10 \mu \mathrm{M})$, respectively ( $\mathrm{n}=5$ independent experiments). (j) HUVECs transfected with control (siCtrl) or PIEZ01-specific siRNA (siPIEZ01) were preactivated with TNFa and were then exposed to PMNs alone, to low flow (1.2 dynes/cm2) alone or to both. After 15 minutes VE-cadherin internalization was determined as described in the Methods $(n=4)$. Shown are mean values \pm s.e.m.; $* P \leq 0.05$; $* * P \leq 0.01$ (unpaired twotailed t- test).
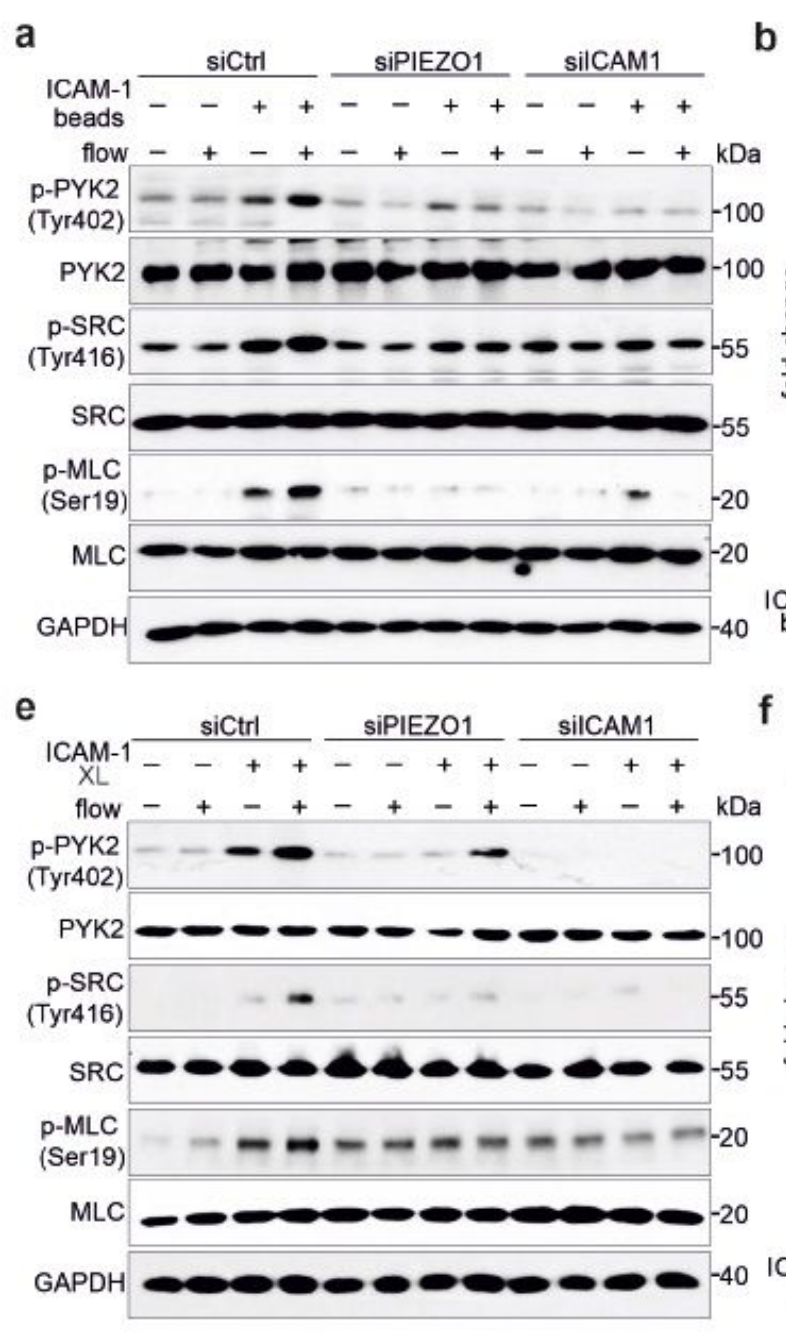

b

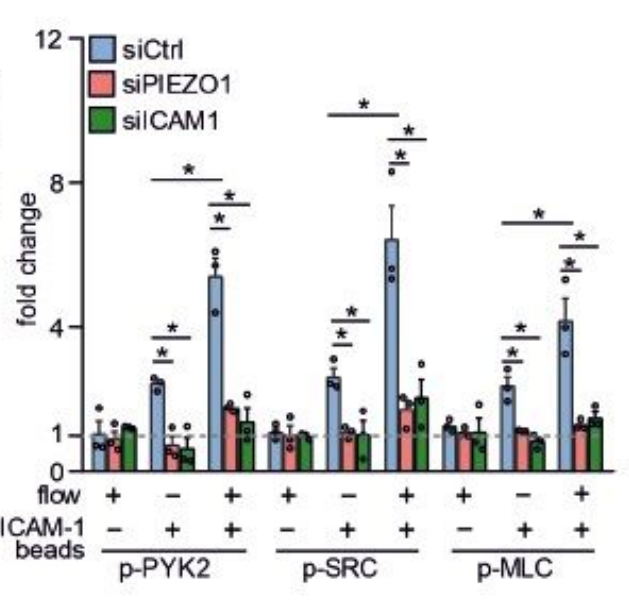

f

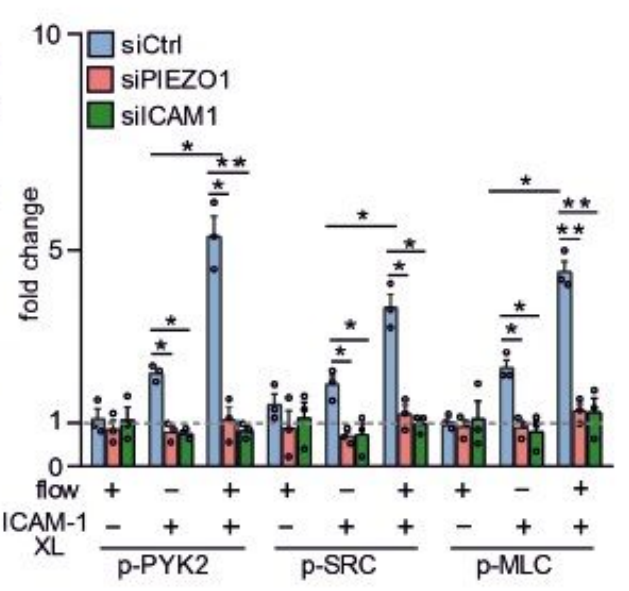

C

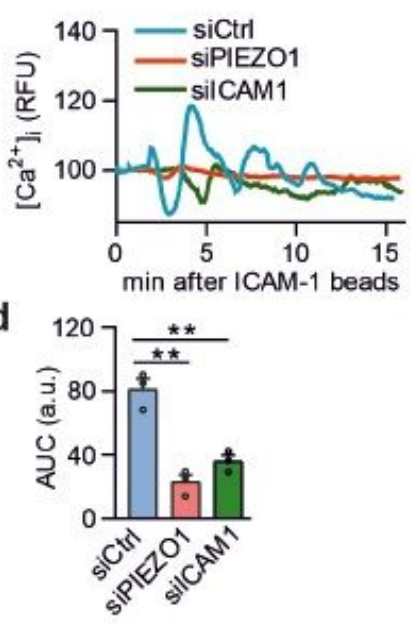

g

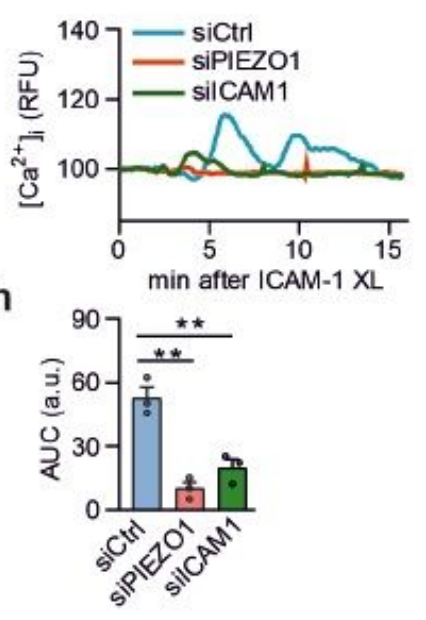

\section{Figure 3}

Endothelial PIEZO1 activation by leukocytes involves ICAM-1 activation and flow. (a-h) TNFa-activated HUVECs transfected with control siRNA (siCtrl) or siRNA directed against ICAM-1 or PIEZO1 were exposed to low flow alone, anti-ICAM-1 antibody beads (ICAM-1 beads) alone or both (a-d) or to low flow alone, anti-ICAM-1 clustering antibodies (ICAM-1 XL) or both (e-h), and immunoblot analysis of total and 
phosphorylated PYK2, SRC and MLC was performed. Immunoblot analysis of GAPDH served as controls. Bar diagrams $(b, f)$ show the densitometric analysis of 3 independent experiments. Alternatively, the free [Ca2+]i was determined after loading of cells with Fluo4 $(\mathrm{c}, \mathrm{g})$. Bar diagrams $(\mathrm{d}, \mathrm{h})$ show the area under the curve (AUC) of the [Ca2+]i-trace from 3 independent experiments (a.u., arbitrary units). Shown are mean values \pm s.e.m.; $* \mathrm{P} \leq 0.05 ; \star \star \mathrm{P} \leq 0.01$ (unpaired two-tailed t-test).

a

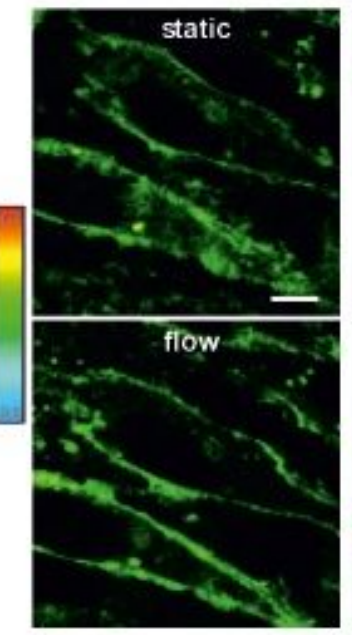

b

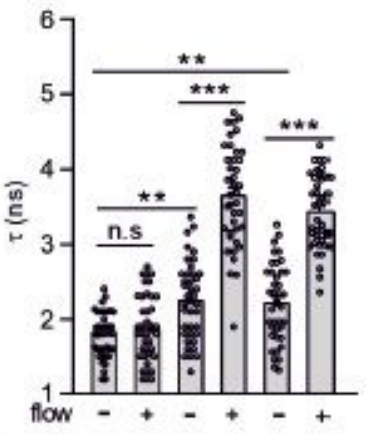

ICAM-1XL - - + + -

ICAM- 1 beads - -+++

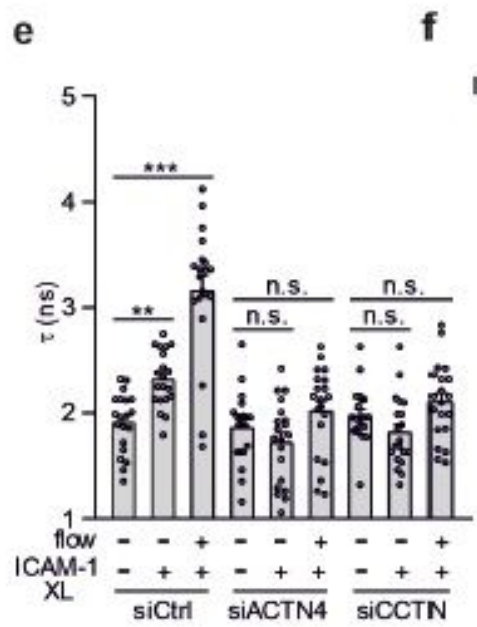

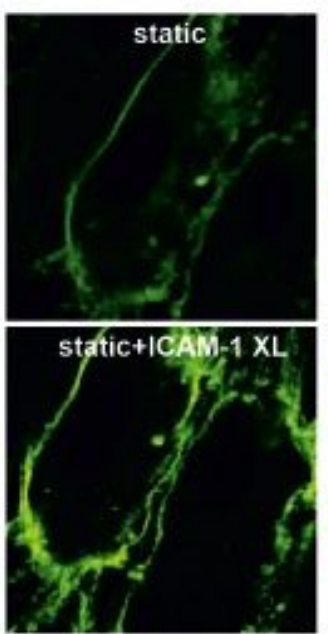

C

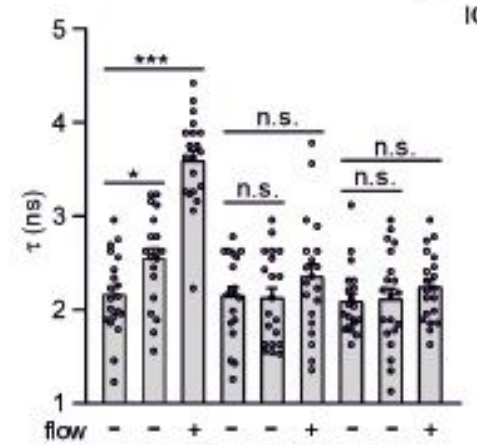

ICAM-1 XL $\frac{-++}{\text { Control }} \frac{-++t}{\text { CytoD }} \frac{-++}{\text { Bleb }}$
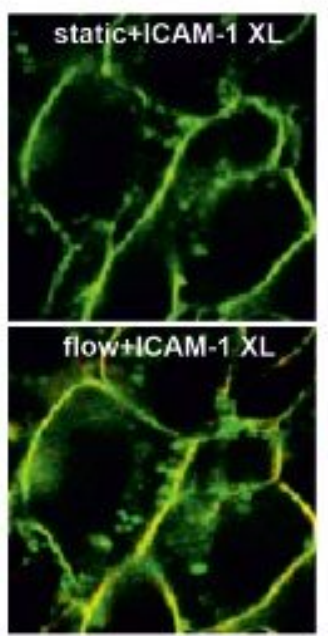

d
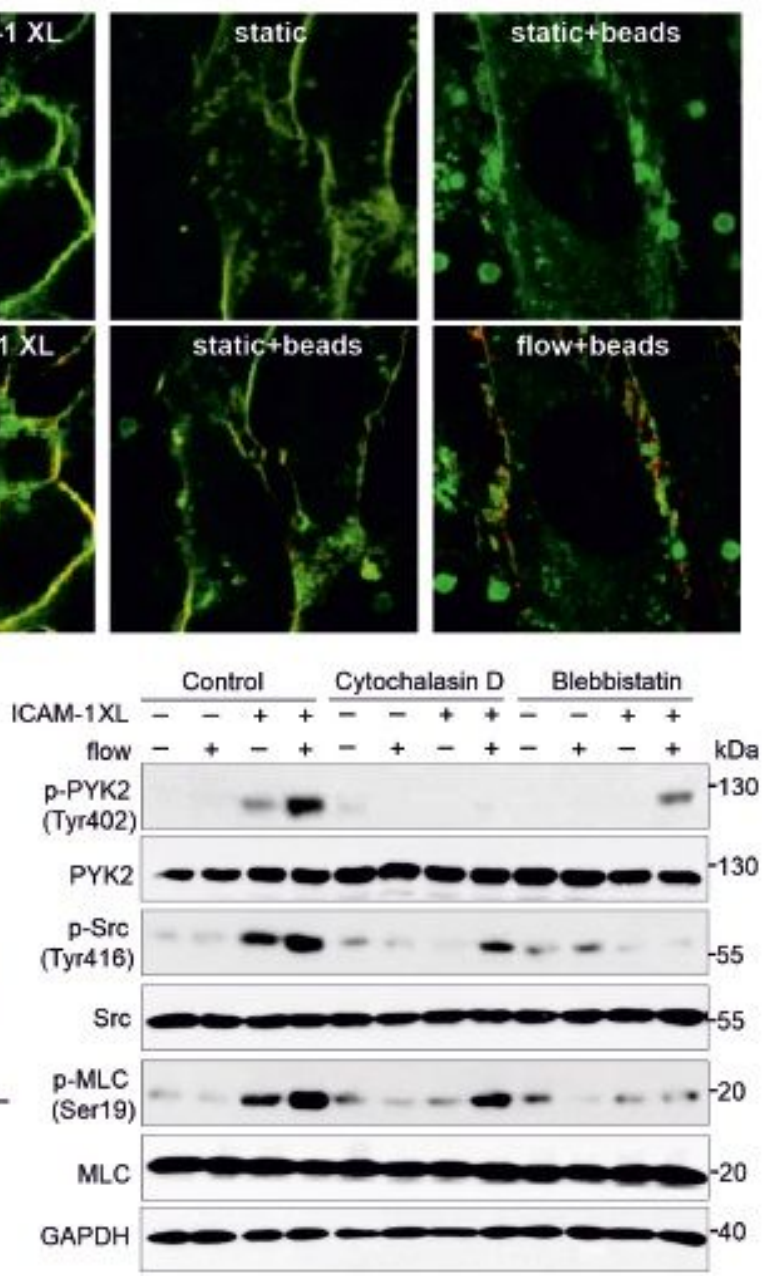

g
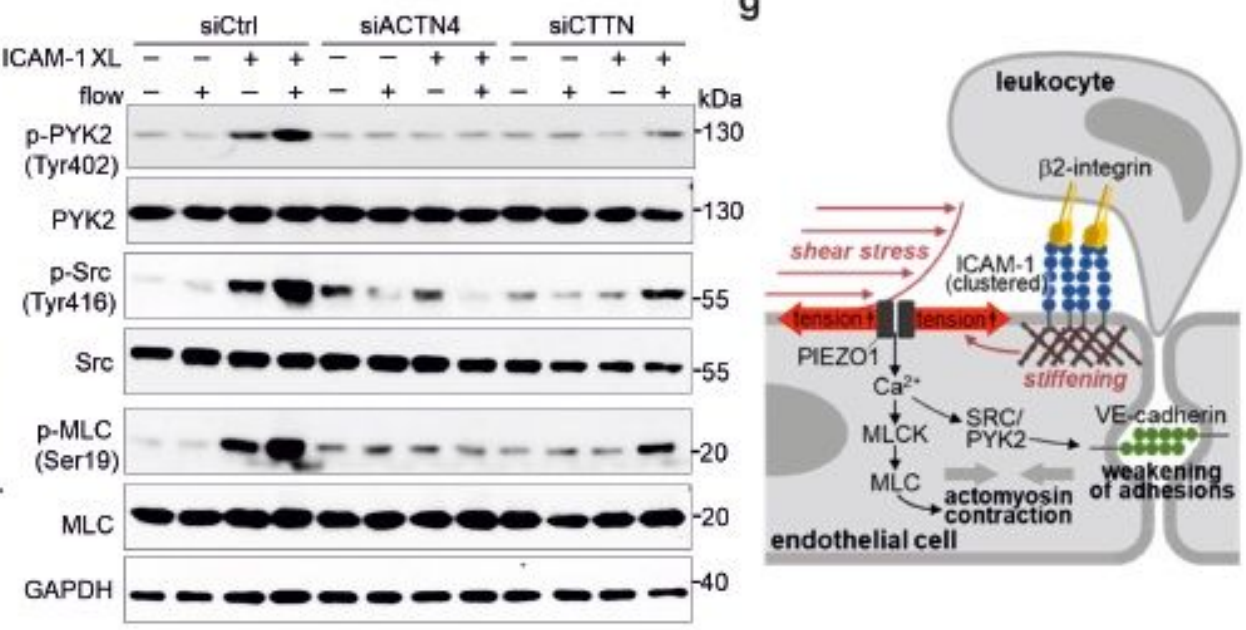

Figure 4 
Flow and ICAM-1 clustering synergistically increase endothelial membrane tension. $(a, b)$ Fluorescence lifetime $\tau 1$ images of FliptR in TNFa-activated HUVECs kept under static conditions or in the presence of low flow (1.2 dynes/cm2) or exposed to anti-ICAM-1 antibody beads or to anti-ICAM-1-crosslinking antibodies (ICAM-1 XL) without or together with low flow. The color bar corresponds to lifetime in nanoseconds (ns). Bar length: $15 \mu \mathrm{m}$. Corresponding lifetime mean values indicating membrane tension are shown in the bar diagram ( $b ; n=40$ measurements from 5 independent experiments). (c-f) HUVECs were preincubated without or with $10 \mu \mathrm{M} 1$ cytochalasin $\mathrm{D}$ (CytoD) or $30 \mu \mathrm{M}$ blebbistatin (Bleb) (c,d) or were transfected with control siRNA (siCtrl) or siRNA directed against the RNA encoding a-actinin-4 3 (siACTN4) or cortactin (siCTTN) (e,f) and were exposed to low flow alone, anti-ICAM-4 1 clustering antibodies (ICAM-1 XL) alone or both, and membrane tension was determined using FliptR (c,e; $n=20$ measurements from independent experiments) or immunoblot analysis of total and phosphorylated PYK2, SRC and MLC was performed (d,f). Bar diagrams show lifetime mean values (c,e). (g) Schematic representation showing how fluid shear stress exerted by the flowing blood and leukocyte-induced ICAM-1 clustering synergistically activate PIEZO1 to induce downstream signaling events resulting in opening of the endothelial barrier. Shown are mean values \pm s.e.m.; ${ }^{*} \leq 0.05 ; * \star P \leq 0.01 ; * \star * P \leq 0.001$ (unpaired twotailed t- test).

\section{Supplementary Files}

This is a list of supplementary files associated with this preprint. Click to download.

- Wangetaleditorialpolicychecklist.pdf 